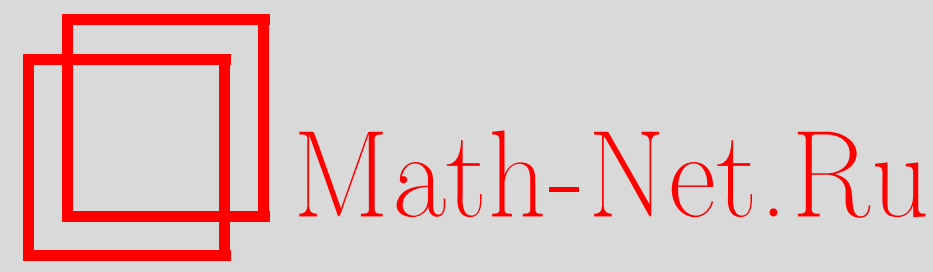

Е. И. Яковлев, Почти главные расслоения, Матем. сб., 1999, том 190, номер 9, 151176

DOI: https://doi.org/10.4213/sm429

Использование Общероссийского математического портала Math-Net.Ru подразумевает, что вы прочитали и согласны с пользовательским соглашением

http://www.mathnet.ru/rus/agreement

Параметры загрузки:

IP : 3.85 .73 .92

26 апреля 2023 г., 16:40:03 
УДК $515.16+515.14$

\author{
Е.И. Яковлев
}

\title{
Почти главные расслоения
}

\begin{abstract}
Исследуется класс $A$ расслоений, обладающих следующими свойствами: каждое расслоение из $A$ представляет собой композицию регулярного накрытия и некоторого главного расслоения (над накрьвающим пространством) с абелевой структурной группой; стандартный слой $G$ этого разложимого расслоения является группой Ли; расслоение обладает атласом с многозначньми функциями перехода со значениями в группе $G$. Класс эквивалентности такого атласа мы называем структурой почти главного расслоения. В работе вычислены группа классов эквивалентности почти главных расслоений с фиксированной базой $B$ и фиксированной структурной группой $G$, ее подгруппа классов эквивалентности главных $G$-расслоений над $B$, а также групшы классов эквивалентности этих расслоений относительно морфизмов категории разложимых расслоений $C$. Найдены база и инвариант почти главного расслоения, не изоморфного главному расслоению даже в категории $C$. Рассмотрены приложения к вариационной задаче с закрепленньми концами для многозначных функционалов.

Библиографол: 6 названий.
\end{abstract}

\section{Введение}

Пусть $\Delta$ - дискретная группа, $T^{k}=(\mathbb{R} / \mathbb{Z})^{k}$ и $T=T^{k} \times \mathbb{R}^{l}$. Любое главное расслоение со структурной группой $G=\Delta \times T$ представляет собой композицию главных $\Delta$ - и $T$-расслоений. Обратное неверно. Выберем регулярное накрытие $\pi: \widetilde{B} \rightarrow B$, проекцию $q: E \rightarrow \widetilde{B}$ некоторого главного $T$-расслоения и положим $p=\pi \circ q$. Тогда $\pi$ - проекция главного расслоения со структурной группой $\Delta=\Pi / \Lambda$, где $\Lambda=\pi_{*}\left(\pi_{1}(\widetilde{B}, \widetilde{b})\right) \subset \Pi=\pi_{1}(B, b), b=\pi(\widetilde{b})$, а $\psi=(E, p, B, G)$ - локально тривиальное расслоение со слоем $G=\Delta \times T$, которое мы будем называть $\Lambda$-разложимьм. Каждая карта $\psi_{U}: U \times G \rightarrow E_{U}$ расслоения $\psi$ порождает действие группы $\Delta$ на $E_{U}$. Однако на пересечениях $E_{V} \cap E_{U}$ эти локальные действия могут не совпадать. Поэтому, вообще говоря, $\psi$ не является главным $G$-расслоением.

В вариационном исчислении оказались полезными разложимые расслоения, обладающие $T$-связностями с проектируемыми на базу формами кривизны. Как показано ниже, такая связность на $E$ обязана быть инвариантной относительно всех локальных действий группы $\Delta$, индуцированных каким-либо атласом расслоения $\psi$. Этот атлас, в свою очередь, должен иметь многозначные функции перехода со значениями в группе $G$. Последнее означает, что для любых его карт $\psi_{U}$ и $\psi_{V}$ с

Работа выполнена при финансовой поддержке Российского фонда фундаментальных исследований (грант № 99-01-00395).

(C) Е.И. Яковлев 1999 
$V \cap U \neq \varnothing$ справедливо равенство $\psi_{U}(a, \alpha, t)=\psi_{V}\left(a, \nu_{V U}(a)+\alpha, \psi_{V U}^{\alpha}(a)+t\right)$, причем $d \psi_{V U}^{\alpha}=d \psi_{V U}$ для всех $\alpha \in \Delta$. Таким образом, многозначная функция перехода ставит в соответствие точке $a \in V \cap U$ не один элемент групшы $G$, а семейство $\left\{\left(\nu_{V U}(a), \psi_{V U}^{\alpha}(a)\right), \alpha \in \Delta\right\} \subset G$. В то же время, ее дифференциал является однозначно определенной 1-формой на $V \cap U$. Класс эквивалентности такого атласа мы называем $\mathscr{A} \mathscr{P} \mathscr{B}$-структурой, а $\Lambda$-разложимое расслоение с $\mathscr{A} \mathscr{P} \mathscr{B}$-структурой почти главным $G \mid \Lambda$-расслоением. Цель настоящей работы - классификация почти главных $G \mid \Lambda$-расслоений над фиксированной базой. Отметим, что главные и ассоциированные с ними векторные расслоения со структурной группой $T^{1}$ изучены в [1], [2].

Рассмотрим почти главные расслоения $\rho$ и $\rho^{\prime}$ со структурной группой $G=\Delta \times T$ и проекциями $p: E \rightarrow B$ и $p^{\prime}: E^{\prime} \rightarrow B$ и гладкое отображение $f: E \rightarrow E^{\prime}$. Если

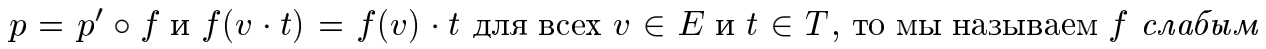
морфизмом почти главных расслоений. Слабый морфизм $f$ называем морфизмом, если карты $\psi_{U}$ и $\psi_{V}^{\prime}$ расслоений $\rho$ и $\rho^{\prime}$ с $V \cap U \neq \varnothing$ также связаны многозначньми функциями перехода, т.е. $f \circ \psi_{U}(a, \alpha, t)=\psi_{V}^{\prime}\left(a, \mu_{V U}(a)+\alpha, \varphi_{V U}^{\alpha}(a)+t\right)$ и $d \varphi_{V U}^{\alpha}=d \varphi V U$

При фиксированном нормальном делителе $\Lambda \subset$ П классы эквивалентности почти главных $G \mid \Lambda$-расслоений над $B$ образуют абелеву группу $\mathscr{A} \mathscr{P} \mathscr{B}(B \mid \Lambda, G)$, а классы эквивалентности главных $G \mid \Lambda$-расслоений - ее подгруппу $\mathscr{P} \mathscr{B}(B \mid \Lambda, G)$. Символами $\mathscr{A} \mathscr{P} \mathscr{B}_{1}(B \mid \Lambda, G)$ и $\mathscr{P} \mathscr{B}_{1}(B \mid \Lambda, G)$ обозначим группы классов эквивалентности тех же расслоений относительно слабых морфизмов.

Сингулярный квадрат $c: I^{2} \rightarrow B, I=[0,1]$, назовем $\Lambda$-квадратом, если все его вершины совпадают с точкой $b$, а гомотопические классы сторон принадлежат $\Lambda$. Сингулярные цепи, состоящие из $\Lambda$-квадратов, будем считать $\Lambda$-цепями. Возникают группы $\Lambda$-циклов, $\Lambda$-границ и $\Lambda$-гомологий $H_{2}(B \mid \Lambda)$. Отождествим $\mathbb{Z}^{k}$ с $\mathbb{Z}^{k} \times 0 \subset \mathbb{R}^{k+l}$. Положим $m=k+l, \Lambda_{0}=\Lambda /[\Lambda, \Lambda]$ и

$\operatorname{Hom}\left(H_{2}(B), H_{2}(B \mid \Lambda) ; \mathbb{R}^{m}, \mathbb{Z}^{k}\right)=\left\{h \in \operatorname{Hom}\left(H_{2}(B), \mathbb{R}^{m}\right): h\left(H_{2}(B \mid \Lambda)\right) \subset \mathbb{Z}^{k}\right\}$

Основными результатами работы являются следующие утверждения.

Teopema 3.6. $\mathscr{A} \mathscr{P} \mathscr{B}(B \mid \Lambda, G) \cong \operatorname{Hom}\left(H_{2}(B), H_{2}(B \mid \Lambda) ; \mathbb{R}^{m}, \mathbb{Z}^{k}\right) \oplus \operatorname{Ext}\left(\Lambda_{0}, \mathbb{Z}^{k}\right)$.

TeOpema 4.5. $\mathscr{P} \mathscr{B}(B \mid \Lambda, G) \cong \operatorname{Hom}\left(H_{2}(B), \mathbb{Z}^{k}\right) \oplus \operatorname{Ext}\left(\Lambda_{0}, \mathbb{Z}^{k}\right)$.

Teopema 5.2. $\mathscr{A} \mathscr{P} \mathscr{B}_{1}(B \mid \Lambda, G) \cong \operatorname{Hom}\left(H_{2}(B \mid \Lambda), \mathbb{Z}^{k}\right)$.

ТЕОРемА 5.4. Пусть гомоморфизмы

$$
\begin{aligned}
& i_{*}: \operatorname{Hom}\left(H_{2}(B), \mathbb{Z}^{k}\right) \rightarrow \operatorname{Hom}\left(H_{2}(B \mid \Lambda), \mathbb{Z}^{k}\right), \\
& j_{*}: \operatorname{Ext}\left(H_{2}(B) / H_{2}(B \mid \Lambda), \mathbb{Z}^{k}\right) \rightarrow \operatorname{Ext}\left(H_{2}(B), \mathbb{Z}^{k}\right)
\end{aligned}
$$

индуцировань короткой точной последовательностью

$$
0 \rightarrow H_{2}(B \mid \Lambda) \stackrel{i}{\rightarrow} H_{2}(B) \stackrel{j}{\rightarrow} H_{2}(B) / H_{2}(B \mid \Lambda) \rightarrow 0 .
$$


Тогда $\mathscr{P} \mathscr{B}_{1}(B \mid \Lambda, G) \cong \operatorname{im} i_{*} u \mathscr{A} \mathscr{P} \mathscr{B}_{1}(B \mid \Lambda, G) / \mathscr{P} \mathscr{B}_{1}(B \mid \Lambda, G) \cong \operatorname{ker} j_{*}$.

С помощью последней формулы доказано существование разложимых расслоений с базой $B=\left(\mathbb{K}^{2} \times S^{1}\right) \#\left(\mathbb{K}^{2} \times S^{1}\right)$, обладающих $\mathscr{A} \mathscr{P} \mathscr{B}$-структурами, но не допускающих структур главных $G$-расслоений. В $\S 7$ рассмотрены приложения почти главных расслоений к вариационным задачам для многозначных функционалов.

Для предложений и теорем используется общая нумерация.

\section{§1. Разложимые расслоения и $\mathscr{A} \mathscr{P} \mathscr{B}$-структуры}

Мы будем пользоваться следующим набором терминов и обозначений. Пусть $\psi=(E, p, B, G)$ - гладкое локально тривиальное расслоение с проекцией $p: E \rightarrow B$ и стандартньп слоем $G, \mathscr{U}$ - открытое покрытие базы $B$. Предположим, что ассоциированньй с $\mathscr{U}$ атлас $\mathscr{A}(\mathscr{U})$ расслоения $\psi$ обладает функциями перехода $\psi_{V U}: V \cap U \rightarrow G$ от карт $\psi_{U}$ к картам $\psi_{V}$ с $V \cap U \neq \varnothing$. Тогда $\mathscr{A}(\mathscr{U})-$ $\mathscr{P} \mathscr{B}$-атлас. Его класс эквивалентности $\mathscr{A}$ представляет собой $\mathscr{P} \mathscr{B}$-структуру. При этом $\xi=(E, p, B, \mathscr{A})$ - главное $G$-расслоение.

Пусть $\pi: \widetilde{B} \rightarrow B$-глад декое регулярное накрытие, $\Pi=\pi_{1}(B, b), \Lambda=\pi_{*}\left(\pi_{1}(\widetilde{B}, \widetilde{b})\right)$ и $\Delta=\Pi / \Lambda$. Тогда $\nu=(\widetilde{B}, \pi, B)$ - главное $\Delta$-расслоение. Класс эквивалентности расслоения $\nu$ однозначно определяется нормальным делителем $\Lambda \subset$ П. Поэтому мы будем назьвать его $\Lambda$-накрытием. Группы $\Pi, \Lambda$ и $\Delta$ договоримся считать аддитивными.

Для сокращения обозначений отождествим касательные пространства к группе $T=(\mathbb{R} / \mathbb{Z})^{k} \times \mathbb{R}^{l}$ в точках $t \in T$ с пространством $\mathbb{R}^{m}, m=k+l$, по изоморфизмам, индуцированным левыми сдвигами $L_{-t}: T \rightarrow T$ и каноническим изоморфизмом $T_{0} T \rightarrow \mathbb{R}^{m}$. Это позволяет считать $\mathbb{R}^{m}$-значными 1-формами дифференциалы гладких отображений в $T$. Определим отображение $e: \mathbb{R}^{m} \rightarrow T$ формулой $e(r)=$ $\left(r_{1}+l_{1} \mathbb{Z}, \ldots, r_{m}+l_{m} \mathbb{Z}\right)$, где $l_{i}=1$ при $i \leqslant k$ и $l_{i}=0$ при $i>k$.

ОПРЕДЕЛЕНИЕ 1.1. Локально тривиальное расслоение $\psi=(E, p, B, G)$ со стандартньм слоем $G=\Delta \times T$ назовем $\Lambda$-разложимым, если найдутся $\Lambda$-накрытие $\nu=(\widetilde{B}, \pi, B)$ и главное $T$-расслоение $\xi=(E, q, \widetilde{B}, \overline{\mathscr{A}})$, для которых $p=\pi \circ q$.

Выберем карты $\nu_{U}: U \times \Delta \rightarrow \widetilde{B}_{U}$ и $\xi_{U}: \widetilde{B}_{U} \times T \rightarrow E_{U}$ расслоений $\nu$ и $\xi$ и положим

$$
\psi_{U}(a, \alpha, t)=\xi_{U}\left(\nu_{U}(a, \alpha), t\right) .
$$

Этим определена карта $\psi_{U}: U \times G \rightarrow E_{U}$ расслоения $\psi$ - композиция карт $\nu_{U}$ и $\xi_{U}$. Предположим, что $\psi_{V}-$ композиция карт $\nu_{V}$ и $\xi_{V}$ и $V \cap U \neq \varnothing$. Рассмотрим функции перехода $\nu_{V U}: V \cap U \rightarrow \Delta$ и $\xi_{V U}: \widetilde{B}_{V} \cap \widetilde{B}_{U} \rightarrow T$. Положив

$$
\psi_{V U}^{\alpha}(a)=\xi_{V U}\left(\nu_{U}(a, \alpha)\right)
$$

определим гладкие отображения $\psi_{V U}^{\alpha}: V \cap U \rightarrow T$. По построению

$$
\psi_{U}(a, \alpha, t)=\psi_{V}\left(a, \nu_{V U}(a)+\alpha, \psi_{V U}^{\alpha}(a)+t\right) .
$$


ОПРЕДЕЛЕНИЕ 1.2. Пусть $\mathscr{A}(\mathscr{U})$ - атлас локально тривиального расслоения $\psi=(E, p, B, G)$ со слоем $G=\Delta \times T, \psi_{U}, \psi_{V} \in \mathscr{A}(\mathscr{U})$ и $V \cap U \neq \varnothing$. Набор отображений $\left\{\left(\nu_{V U}, \psi_{V U}^{\alpha}\right): V \cap U \rightarrow G, \alpha \in \Delta\right\}$, удовлетворяющих равенству (1.3) при всех $a \in V \cap U, \alpha \in \Delta$ и $t \in T$, мы будем называть псевдофункиией перехода от карты $\psi_{U}$ к карте $\psi_{V}$.

Итак, $\Lambda$-разложимое расслоение обладает атласом с системой псевдофункций перехода. Верно и обратное. Если $\mathscr{A}(\mathscr{U})$ - атлас расслоения $\psi$ с псевдофункциями перехода $\left\{\left(\nu_{V U}, \psi_{V U}^{\alpha}\right)\right\}$, то в силу (1.3) набор $\left\{\nu_{V U}\right\}$ является коциклом. Поэтому существует главное $\Delta$-расслоение $\nu=(\widetilde{B}, \pi, B)$ с атласом $\widetilde{\mathscr{A}}(\mathscr{U})$ и функциями перехода $\left\{\nu_{V U}\right\}$. Для карт $\psi_{U} \in \mathscr{A}(\mathscr{U})$ и $\nu_{U} \in \widetilde{\mathscr{A}}(\mathscr{U})$ положим $q\left(\psi_{U}(a, \alpha, t)\right)=$ $\nu_{U}(a, \alpha)$. Этим корректно определено гладкое отображение $q: E \rightarrow \widetilde{B}$, причем $p=\pi \circ q$. Формула (1.1) определяет карту $\xi_{U}: \widetilde{B}_{U} \times T \rightarrow E_{U}$. При $U, V \in \mathscr{U}$ и $V \cap U \neq \varnothing$ формулой (1.2) определена функция перехода $\xi_{V U}$ от карты $\xi_{U}$ к карте $\xi_{V}$. Это значит, что $\overline{\mathscr{A}}(\mathscr{U})=\left\{\xi_{U}, U \in \mathscr{U}\right\}$ - атлас главного $T$-расслоения $\xi=(E, q, \widetilde{B}, \bar{A})$.

ОПРЕДЕЛЕНИЕ 1.3. Пусть $\psi=(E, p, B, G)-\Lambda$-разложимое расслоение, $\mathscr{A}(\mathscr{U})$ - его атлас, $\psi_{U}, \psi_{V} \in \mathscr{A}(\mathscr{U})$ и $\left\{\left(\nu_{V U}, \psi_{V U}^{\alpha}\right), \alpha \in \Delta\right\}$ - псевдофункция перехода. Если дифференциалы $d \psi_{V U}^{\alpha}$ не зависят от $\alpha$, то мы будем полагать $d \psi_{V U}=d \psi_{V U}^{\alpha}$. При этом набор $\left\{\left(\nu_{V U}, \psi_{V U}^{\alpha}\right)\right\}$ будем называть многозначной функиией перехода

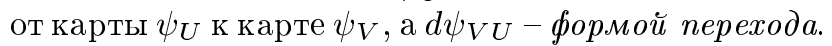

ОПРЕДЕЛЕНИЕ 1.4. Допустим, что $\mathscr{A}(\mathscr{U})$ - атлас $\Lambda$-разложимого расслоения и для любых пар карт $\psi_{U}, \psi_{V} \in \mathscr{A}(\mathscr{U})$ с $V \cap U \neq \varnothing$ сушествует форма перехода $d \psi_{V U}$. Тогда $\mathscr{A}(\mathscr{U})-\mathscr{A} \mathscr{P} \mathscr{B}$ - атлас. Два $\mathscr{A} \mathscr{P} \mathscr{B}$-атласа мы считаем эквивалентными, если их объединение также является $\mathscr{A} \mathscr{P} \mathscr{B}$-атласом. Класс эквивалентности $\mathscr{A} \mathscr{P} \mathscr{B}$-атласа назьваем $\mathscr{A} \mathscr{P} \mathscr{B}$-структурой. Если $\Lambda$-разложимое расслоение $\psi=(E, p, B, G)$ обладает $\mathscr{A} \mathscr{P} \mathscr{B}$-структурой $\mathscr{A}$, то $\rho=(E, p, B, \mathscr{A})$ - почти әлавное $G \mid \Lambda$-расслоение.

Зафиксируем нормальный делитель $\Lambda \subset \Pi, \Lambda$-накрытие $\nu=(\widetilde{B}, \pi, B)$, простое покрытие $\mathscr{U}$ базы $B$, а также атлас $\tilde{\mathscr{A}}(\mathscr{U})$ накрытия $\nu$ с функциями перехода $\left\{\nu_{V U}\right\}$. Тогда $\nu_{V U} \equiv$ const, любое почти главное $G \mid \Lambda$-расслоение над $B$ представимо в виде композиции данного накрытия $\nu$ и некоторого главного $T$-расслоения над $\widetilde{B}$, а соответствующая $\mathscr{A} \mathscr{P} \mathscr{B}$-структура содержит атлас $\mathscr{A}(\mathscr{U})$ с многозначными функциями перехода вида $\left\{\left(\nu_{V U}, \psi_{V U}^{\alpha}\right)\right\}$. В тех случаях, когда набор $\left\{\nu_{V U}\right\}$ явно не используется, мы будем сокращать обозначение этой функции до символа $\left\{\psi_{V U}^{\alpha}\right\}$.

ОПРЕДЕЛЕНИЕ 1.5. Рассмотрим $\Lambda$-разложимые расслоения $\psi=(E, p, B, G)$, $\psi^{\prime}=\left(E^{\prime}, p^{\prime}, B, G\right)$ и гладкое отображение $f: E \rightarrow E^{\prime}$. По определению на $E$ и $E^{\prime}$ определены правые действия группы $T$. Если $p^{\prime} \circ f=p$ и $f(v \cdot t)=f(v) \cdot t$ для всех $v \in E$ и $t \in T$, то мы будем говорить, что $f: \psi \rightarrow \psi^{\prime}-$ морфизм $\Lambda$-разложсимых расслоений (над $B$ ). Если расслоения $\psi$ и $\psi^{\prime}$ обладают $\mathscr{A} \mathscr{P} \mathscr{B}$-структурами $\mathscr{A}$ и $\mathscr{A}^{\prime}$, то $f$ будет называться слабым морфизмом почти главных $G \mid \Lambda$-расслоений $\rho=(E, p, B, \mathscr{A})$ и $\rho^{\prime}=\left(E^{\prime}, p^{\prime}, B, \mathscr{A}^{\prime}\right)$. 
Заметим, что упомянутые морфизмы всегда являются изоморфизмами [3; гл. 4 , теорема 3.2]. Согласно замечанию после определения 1.4 можно считать, что $p=\pi \circ q$ и $p^{\prime}=\pi \circ q^{\prime}$, где $\xi=(E, q, \widetilde{B}, \overline{\mathscr{A}})$ и $\xi^{\prime}=\left(E^{\prime}, q^{\prime}, \widetilde{B}, \overline{\mathscr{A}}^{\prime}\right)$ - главные $T$-расслоения, а слабый морфизм представляет собой морфизм главных расслоений $f: \xi \rightarrow \xi^{\prime}$ над $\widetilde{B}$. Выберем атласы $\mathscr{A}(\mathscr{U}) \in \mathscr{A}$ и $\mathscr{A}^{\prime}(\mathscr{U}) \in \mathscr{A}^{\prime}$. Карты $\psi_{U} \in \mathscr{A}(\mathscr{U})$ и $\psi_{V}^{\prime} \in \mathscr{A}^{\prime}(\mathscr{U})$ являются композициями карт $\nu_{U}$ и $\nu_{V}$ накрытия $\nu$ и карт $\xi_{U}$ и $\xi_{V}^{\prime}$ расслоений $\xi$ и $\xi^{\prime}$. Для последних имеются функции перехода $\zeta_{V U}: \widetilde{B}_{V} \cap \widetilde{B}_{U} \rightarrow T$. Положим $\varphi_{V U}^{\alpha}(a)=\zeta_{V U}\left(\nu_{U}(a, \alpha)\right)$. Отображение $\varphi_{V U}^{\alpha}: V \cap U \rightarrow T$ удовлетворяет равенству

$$
f \circ \psi_{U}(a, \alpha, t)=\psi_{V}^{\prime}\left(a, \nu_{V U}+\alpha, \varphi_{V U}^{\alpha}(a)+t\right) .
$$

ОПРЕДЕЛЕНИЕ 1.6. Набор $\left\{\varphi_{V U}^{\alpha}, \alpha \in \Delta\right\}$ удовлетворяющих равенству (1.4) отображений $\varphi_{V U}^{\alpha}: V \cap U \rightarrow T$ далее будет назьваться псевдофункиией перехода от карты $\psi_{U}$ к карте $\psi_{V}^{\prime}$ при морфизме $f$.

ОПРЕДЕЛЕНИЕ 1.7. Слабый морфизм $f: \rho \rightarrow \rho^{\prime}$ назовем морфизмом почти главных $G \mid \Lambda$-расслоений $\rho=(E, p, B, \mathscr{A})$ и $\rho^{\prime}=\left(E^{\prime}, p^{\prime}, B, \mathscr{A}^{\prime}\right)$, если псевдофункции перехода (при морфизме $f$ ) от карт атласов $\mathscr{A} \mathscr{P} \mathscr{B}$-структуры $\mathscr{A}$ к картам атласов $\mathscr{A} \mathscr{P} \mathscr{B}$-структуры $\mathscr{A}^{\prime}$ являются многозначными функциями перехода, т.е. корректно определяют формы перехода $d \varphi_{V U}=d \varphi_{V U}^{\alpha}$.

Нетрудно показать, что слабый морфизм $f: \rho \rightarrow \rho^{\prime}$ почти главных $G \mid \Lambda$-расслоений является морфизмом тогда и только тогда, когда они обладают такими $\mathscr{A} \mathscr{P} \mathscr{B}$-атласами $\mathscr{A}(\mathscr{U})$ и $\mathscr{A}^{\prime}(\mathscr{U})$, что для любых карт $\psi_{U} \in \mathscr{A}(\mathscr{U})$ и $\psi_{U}^{\prime} \in \mathscr{A}^{\prime}(\mathscr{U})$ справедливо равенство $f \circ \psi_{U}=\psi_{U}^{\prime}$.

ОПРЕДЕЛЕНИЕ 1.8. Пусть $\psi_{U}: U \times G \rightarrow E_{U}$ - карта $\Lambda$-разложимого расслоения $\psi=(E, p, B, G)$. Для точки $a \in U$ и элементов $\alpha, \gamma \in \Delta$ и $t \in T$ положим $\delta_{U}\left(\psi_{U}(a, \alpha, t), \gamma\right)=\psi_{U}(a, \alpha+\gamma, t)$. Этим определено правое действие $\delta_{U}: E_{U} \times \Delta \rightarrow E_{U}$. Его будем называть локальным действием группы $\Delta$ на $E$, индуцированным картой $\psi_{U}$.

Рассмотрим $\Lambda$-разложимые расслоения $\psi=(E, p, B, G)$ и $\psi^{\prime}=\left(E^{\prime}, p^{\prime}, B, G\right)$ и морфизм $f: \psi \rightarrow \psi^{\prime}$. Тогда в силу (1.4) для любых карт $\psi_{U} \in \mathscr{A}(\mathscr{U})$ и $\psi_{V}^{\prime} \in \mathscr{A}^{\prime}(\mathscr{U})$ с псевдофункцией перехода $\left\{\varphi_{V U}^{\alpha}, \alpha \in \Delta\right\}$ при морфизме $f$ индуцированные ими локальные действия $\delta_{U}: E_{U} \times \Delta \rightarrow E_{U}$ и $\delta_{V}^{\prime}: E_{V}^{\prime} \times \Delta \rightarrow E_{V}^{\prime}$ удовлетворяют равенству

$$
f \circ \delta_{U}(v, \gamma)=\delta_{V}^{\prime}(f(v), \gamma) \cdot\left(\varphi_{V U}^{\alpha+\gamma}-\varphi_{V U}^{\alpha}\right)(a)
$$

где $v=\psi_{U}(a, \alpha, t)$ и $\gamma \in \Delta$. При этом $\left\{\varphi_{V U}^{\alpha}\right\}-$ многозначная функция перехода тогда и только тогда, когда для каждой компоненты связности $K$ пересечения $V \cap U$ существует такой элемент $\theta_{K}^{\alpha}(\gamma) \in T$, что $f \circ \delta_{U}(v, \gamma)=\delta_{V}^{\prime}(f(v), \gamma) \cdot \theta_{K}^{\alpha}(\gamma)$ при всех $v \in E_{K}$.

Согласно сказанному определения $\mathscr{A} \mathscr{P} \mathscr{B}$-атласа, $\mathscr{A} \mathscr{P} \mathscr{B}$-структуры и морфизма почти главных $G \mid \Lambda$-расслоений очевидным образом переформулируются в терминах локальных действий группы $\Delta$. В частности, слабый морфизм $f: \rho \rightarrow \rho^{\prime}$ почти главных $G \mid \Lambda$-расслоений является морфизмом тогда и только тогда, когда он эквивариантен относительно локальных действий групшы $\Delta$, индуцированных некоторьми атласами расслоений $\rho$ и $\rho^{\prime}$. 


\section{§2. Связности}

Рассмотрим морфизм $f: \psi \rightarrow \psi^{\prime} \Lambda$-разложимых расслоений $\psi=(E, p, B, G)$ и $\psi^{\prime}=\left(E^{\prime}, p^{\prime}, B, G\right)$ и $T$-связности $H$ на $E$ и $H^{\prime}$ на $E^{\prime}$, связанные формулой $H_{f(v)}^{\prime}=d f\left(H_{v}\right)$. Выберем карты $\psi_{U}$ и $\psi_{V}^{\prime}$ расслоений $\psi$ и $\psi^{\prime}$ соответственно и индуцированные ими локальные действия $\delta_{U}$ и $\delta_{V}^{\prime}$ группы $\Delta$. Предположим, что $V \cap U \neq \varnothing$.

ПРЕДЛОЖЕНИЕ 2.1. Если связность Н инвариантна относительно действия $\delta_{U}$, то $H^{\prime}$ инвариантна на $E_{V \cap U}^{\prime}$ относительно $\delta_{V}^{\prime}$ тогда и только тогда, когда существует форма перехода $d \varphi_{V U}$ от карть $\psi_{U} \kappa$ карте $\psi_{V}^{\prime}$ при морфизме $f$.

ДокАЗАТЕльство. Предположим сначала, что связность $H^{\prime}$ инвариантна на $E_{V \cap U}^{\prime}$ относительно действия $\delta_{V}^{\prime}$. Выберем элементы $\alpha, \gamma \in \Delta$, точки $a_{0}, a_{1} \in$ $V \cap U$ и путь $x: I \rightarrow V \cap U$ с началом $x(0)=a_{0}$ и концом $x(1)=a_{1}$. Для некоторого $t_{0} \in T$ положим $v_{0}=\psi_{U}\left(a_{0}, \alpha, t_{0}\right)$. Рассмотрим горизонтальный относительно связности $H$ лифт $y$ пути $x$ с начальной точкой $y(0)=v_{0}$. Тогда найдется такое $t_{1} \in T$, что $y(1)=v_{1}=\psi_{U}\left(a_{1}, \alpha, t_{1}\right)$. Положим $y_{U}(s)=f\left(\delta_{U}(y(s), \gamma)\right)$ и $y_{V}(s)=\delta_{V}^{\prime}(f(y(s)), \gamma)$ для $s \in I$. Пути $y_{U}: I \rightarrow E^{\prime}$ и $y_{V}: I \rightarrow E^{\prime}$ горизонтальны относительно связности $H^{\prime}$. Кроме того, $p^{\prime} \circ y_{U}=p^{\prime} \circ y_{V}$. Поэтому сушествует элемент $\theta \in T$, при всех $s \in I$ удовлетворяющий равенству $y_{U}(s)=y_{V}(s) \cdot \theta$. В частности, $f\left(\delta_{U}\left(v_{i}, \gamma\right)\right)=\delta_{V}^{\prime}\left(f\left(v_{i}\right), \gamma\right) \cdot \theta, i=0,1$. Отсюда и из $(1.5)$ следует, что $\left(\varphi_{V U}^{\alpha+\gamma}-\varphi_{V U}^{\alpha}\right)\left(a_{i}\right)=\theta$.

Допустим далее, что для $i=0,1$ и произвольных $\alpha, \gamma \in \Delta$ верно предыдушее равенство. Положим $r=-\theta$ и $R_{r}\left(v^{\prime}\right)=v^{\prime} \cdot r$ для $v^{\prime} \in E^{\prime}$. Тогда согласно (1.5) $R_{r} \circ f \circ\left(\delta_{U}\right)_{\gamma}=\left(\delta_{V}^{\prime}\right)_{\gamma} \circ f$ на $E_{V \cap U}$. Для доказательства инвариантности $H^{\prime}$ относительно $\delta_{V}^{\prime}$ осталось продифференцировать последнее равенство и воспользоваться инвариантностью $H$ относительно $\delta_{U}$ и инвариантностью $H^{\prime}$ относительно действия группы $T$.

ПРЕДЛОЖЕНИЕ 2.2. Для того чтобъ на пространстве $\Lambda$-разложимого расслоения $\psi=(E, p, B, G)$ существовала T-связность, инвариантная относительно всех локальных действий группь $\Delta$, индучированных атласом $\mathscr{A}(\mathscr{U})$, необходимо и достаточно, чтобы последний был $\mathscr{A} \mathscr{P} \mathscr{B}$-атласом.

ДокАЗАТЕльство. Пусть $T$-связность $H$ на $E$ инвариантна относительно действий $\delta_{U}$, индуцированных картами $\psi_{U} \in \mathscr{A}(\mathscr{U})$. Из предложения 2.1 при $\psi^{\prime}=\psi$, $H^{\prime}=H$ и $f=$ id следует, что $\mathscr{A}(\mathscr{U})-\mathscr{A} \mathscr{P} \mathscr{B}$-атлас.

С другой стороны, для $\mathscr{A} \mathscr{P} \mathscr{B}$-атласа $\mathscr{A}(\mathscr{U})$ и каждого $U \in \mathscr{U}$ сужение $p_{U}: E_{U} \rightarrow U$ является главным расслоением со структурной группой $G=\Delta \times T$. Выберем $G$-связность на $E_{U}$ с формой связности $\omega^{U}$. Символом $\widehat{\omega}^{U}$ обозначим продолжение последней на $E$. На $B$ рассмотрим разбиение единицы $\left\{\chi^{U}, U \in \mathscr{U}\right\}$ с носителями $\operatorname{supp} \chi^{U} \subset U$. Положим $\widehat{\chi}^{U}=\chi^{U} \circ p$ и $\omega=\sum_{U \in \mathscr{U}} \widehat{\chi}^{U} \widehat{\omega}^{U}$. Этим на $E$ определена форма $\omega$ некоторой $T$-связности $H$. По построению и по предложению 2.1 каждая форма $\widehat{\omega}^{U}$ инвариантна относительно всех действий $\delta_{V}, V \in \mathscr{U}$. Следовательно, форма $\omega$ и связность $H$ инвариантны относительно локальных действий группы $\Delta$, индуцированных атласом $\mathscr{A}(\mathscr{U})$. 
ОПРЕДЕЛЕНИЕ 2.1. Пусть $\psi=(E, p, B, G)-\Lambda$-разложимое расслоение и $H$ $T$-связность на $E$, инвариантная относительно локальных действий группы $\Delta$, индуцированных некоторьм атласом $\mathscr{A}(\mathscr{U})$. Согласно предложению 2.2 атлас $\mathscr{A}(\mathscr{U})$ является $\mathscr{A} \mathscr{P} \mathscr{B}$-атласом. Если $H$ инвариантна относительно локальных действий группы $\Delta$, индуцированных другим $\mathscr{A} \mathscr{P} \mathscr{B}$-атласом, то по предложению 2.1 последний эквивалентен атласу $\mathscr{A}(\mathscr{U})$. Поэтому $H$ будет называться $G$-связностью относительно содержащей этот атлас $\mathscr{A} \mathscr{P} \mathscr{B}$-структуры $\mathscr{A}$ или $G$-связностью на пространстве почти главного $G \mid \Lambda$-расслоения $\rho=(E, p, B, \mathscr{A})$.

Рассмотрим почти главные $G \mid \Lambda$-расслоения $\rho=(E, p, B, \mathscr{A})$ и $\rho^{\prime}=\left(E^{\prime}, p^{\prime}, B, \mathscr{A}^{\prime}\right)$, $G$-связность $H$ на $E$ и слабый морфизм $f: \rho \rightarrow \rho^{\prime}$. Для каждой точки $v^{\prime} \in E^{\prime}$ положим $H_{v^{\prime}}^{\prime}=d f\left(H_{f^{-1}\left(v^{\prime}\right)}\right)$. Этим определена $T$-связность $H^{\prime}$ на $E^{\prime}$. В силу предложения 2.1 она является $G$-связностью тогда и только тогда, когда $f: \rho \rightarrow \rho^{\prime}$ - морфизм почти главных расслоений.

ОПРЕДЕЛЕНИЕ 2.2. Пусть $\Omega$ - форма кривизны $T$-связности $H$ на пространстве $\Lambda$-разложимого расслоения $\psi=(E, p, B, G)$. Мы будем говорить, что она $n p o е к-$ тируема на базу $B$, если сушествует замкнутая 2-форма $\Phi$ со значениями в $\mathbb{R}^{m}$, для которой $\Omega=p^{*} \Phi$.

ПРЕДЛОЖЕНИЕ 2.3. Форма кривизны $\Omega$ некоторой T-связности $H$ на пространстве $\Lambda$-разложимого расслоения $\psi=(E, p, B, G)$ проектируема на базу $B$ в том и только том случае, если существует $\mathscr{A} \mathscr{P} \mathscr{B}$-структура $\mathscr{A}$, относительно которой $H$ является $G$-связностью.

ДоказАтЕЛЬство. Рассмотрим атлас $\mathscr{A}(\mathscr{U})$ расслоения $\psi$, индуцированное картой $\psi_{U} \in \mathscr{A}(\mathscr{U})$ локальное действие $\delta_{U}: E_{U} \times \Delta \rightarrow E_{U}$ и индуцированное структурой накрытия $\nu$ действие $\delta: \widetilde{B} \times \Delta \rightarrow \widetilde{B}$. В силу (1.1) для всех $\gamma$ имеет место равенство $q \circ\left(\delta_{U}\right)_{\gamma}=\delta_{\gamma} \circ q$.

Предположим, что $H-G$-связность относительно $\mathscr{A} \mathscr{P} \mathscr{B}$-структуры $\mathscr{A}$. На $\widetilde{B}$ существует замкнутая $\mathbb{R}^{m}$-значная 2-форма $\widetilde{\Phi}$, удовлетворяющая равенству $\Omega=q^{*} \widetilde{\Phi}$. Согласно предыдушему равенству $q^{*}\left(\delta_{\gamma}^{*} \widetilde{\Phi}\right)=\left(\delta_{U}\right)_{\gamma}^{*} \Omega=\Omega=q^{*} \widetilde{\Phi}$. При этом $\delta_{\gamma}^{*} \widetilde{\Phi}=\widetilde{\Phi}$ и потому найдется замкнутая 2 -форма $\Phi$ на $B$, для которой $\widetilde{\Phi}=\pi^{*} \Phi$. В результате: $\Omega=q^{*}\left(\pi^{*} \Phi\right)=p^{*} \Phi$.

Пусть далее дано, что $\Omega=p^{*} \Phi$. Тогда из равенства $p \circ\left(\delta_{U}\right)_{\gamma}=p$ следует, что $\left(\delta_{U}\right)_{\gamma}^{*} \Omega=\Omega$. Для произвольного $\alpha \in \Delta$ положим

$$
\sigma_{U}^{\alpha}(a)=\psi_{U}(a, \alpha, 0), \quad \omega_{U}^{\alpha}=\left(\sigma_{U}^{\alpha}\right)^{*} \omega
$$

Так как $\sigma_{U}^{\gamma^{\prime}}=\left(\delta_{U}\right)_{\gamma^{\prime}} \circ \sigma_{U}^{0}$, то

$$
\omega_{U}^{\gamma^{\prime}}=\left(\sigma_{U}^{0}\right)^{*}\left(\delta_{U}\right)_{\gamma^{\prime}}^{*} \omega
$$

Поэтому $d\left(\omega_{U}^{\gamma^{\prime}}-\omega_{U}^{0}\right)=0$, откуда в силу простоты покрытия $\mathscr{U}$ следует существование гладкой функции $f_{U}^{\gamma^{\prime}}: U \rightarrow \mathbb{R}^{m}$, удовлетворяюшей равенству 
$\omega_{U}^{\gamma^{\prime}}=\omega_{U}^{0}+d f_{U}^{\gamma^{\prime}}$. Но тогда $\omega_{U}^{\alpha+\gamma}-\omega_{U}^{\alpha}=d f_{U}^{\alpha+\gamma}-d f_{U}^{\alpha}$. С другой стороны, в силу $(2.1) \omega_{U}^{\alpha+\gamma}-\omega_{U}^{\alpha}=\left(\sigma_{U}^{\alpha}\right)^{*}\left(\left(\delta_{U}\right)_{\gamma}^{*} \omega-\omega\right)$. Таким образом,

$$
\left(\left(\delta_{U}\right)_{\gamma}^{*} \omega-\omega\right) \circ d \sigma_{U}^{\alpha}=d f_{U}^{\alpha+\gamma}-d f_{U}^{\alpha}
$$

Формула $\widehat{\psi}_{U}(a, \alpha, t)=\psi_{U}\left(a, \alpha, t-e \circ f_{U}^{\alpha}(a)\right)$ определяет карту $\widehat{\psi}_{U}: U \times G \rightarrow$ $E_{U}$ расслоения $\psi$. Индуцированное ею действие $\widehat{\delta}_{U}: E_{U} \times \Delta \rightarrow E_{U}$ связано с действием $\delta_{U}$ формулой

$$
\left(\delta_{U}\right)_{\gamma}(v)=\left(\widehat{\delta}_{U}\right)_{\gamma}(v) \cdot e \circ\left(f_{U}^{\alpha+\gamma}-f_{U}^{\alpha}\right) \circ p(v)
$$

Поэтому

$$
\omega \circ d\left(\delta_{U}\right)_{\gamma}=\omega \circ d\left(\widehat{\delta}_{U}\right)_{\gamma}+\left(d f_{U}^{\alpha+\gamma}-d f_{U}^{\alpha}\right) \circ d p
$$

и, следовательно, $\left(\left(\delta_{U}\right)_{\gamma}^{*} \omega-\left(\widehat{\delta}_{U}\right)_{\gamma}^{*} \omega\right) \circ d \sigma_{U}^{\alpha}=d f_{U}^{\alpha+\gamma}-d f_{U}^{\alpha}$. В силу (2.3) отсюда вытекает, что $\left(\left(\widehat{\delta}_{U}\right)_{\gamma}^{*} \omega-\omega\right) \circ d \sigma_{U}^{\alpha}=0$.

Рассмотрим точку $v \in E_{U}^{\alpha}$ и вектор $X \in T_{v} E$. Положим $X_{0}=d \sigma_{U}^{\alpha} \circ d p(X)$ и $X_{1}=X-X_{0}$. Тогда по доказанному $\left(\left(\widehat{\delta}_{U}\right)_{\gamma}^{*} \omega-\omega\right)\left(X_{0}\right)=0$. Так как вектор $X_{1}$ вертикален, то найдется элемент $r \in \mathbb{R}^{m}$, для которого $X_{1}=\left.\frac{d}{d s}(v \cdot e(s r))\right|_{s=0}$. При этом $\omega\left(X_{1}\right)=r$. По определению дифференциала гладкого отображения

$$
d\left(\widehat{\delta}_{U}\right)_{\gamma}\left(X_{1}\right)=\left.\frac{d}{d s}\left(\widehat{\delta}_{U}(v \cdot e(s r), \gamma)\right)\right|_{s=0}=\left.\frac{d}{d s}\left(\widehat{\delta}_{U}(v, \gamma) \cdot e(s r)\right)\right|_{s=0} .
$$

Но тогда $\omega\left(d\left(\widehat{\delta}_{U}\right)_{\gamma}\left(X_{1}\right)\right)=r$ и, следовательно, $\left(\left(\widehat{\delta}_{U}\right)_{\gamma}^{*} \omega-\omega\right)\left(X_{1}\right)=r-r=0$. Этим доказано, что $\left(\left(\widehat{\delta}_{U}\right)_{\gamma}^{*} \omega-\omega\right)(X)=0$ для произвольного $X \in T_{v} E$. Следовательно, форма $\omega$, а вместе с нею и связность $H$ инвариантны относительно локальных действий группы $\Delta$, индуцированных атласом $\widehat{\mathscr{A}}(\mathscr{U})=\left\{\widehat{\psi}_{U}, U \in \mathscr{U}\right\}$. Согласно предложению $2.2 \widehat{\mathscr{A}}(\mathscr{U})-\mathscr{A} \mathscr{P} \mathscr{B}$-атлас, а $H-G$-связность относительно содержащей построенный атлас $\mathscr{A} \mathscr{P} \mathscr{B}$-структуры.

ОПРЕДЕЛЕНИЕ 2.3. Пусть $\rho=(E, p, B, \mathscr{A})$ - почти главное $G \mid \Lambda$-расслоение и $\omega$ - форма $G$-связности $H$ на $E$. Выберем атлас $\mathscr{A}(\mathscr{U}) \in \mathscr{A}$ и карту $\psi_{U} \in \mathscr{A}(\mathscr{U})$. Формулами (2.1) определим локальные сечения $\sigma_{U}^{\alpha}: U \rightarrow E$ расслоения $\rho$ и 1-формы $\omega_{U}^{\alpha}$ на $U$. В силу $(2.2)$ и инвариантности $H$ относительно действия $\delta_{U}$ эти формы одинаковы при всех $\alpha \in \Delta$. Поэтому мы будем полагать $\omega_{U}=\omega_{U}^{\alpha}$ и называть $\omega_{U}$ локальной формой связности $H$.

ПреДЛОЖЕНИЕ 2.4. Пусть $\rho=(E, p, B, \mathscr{A})$ u $\rho^{\prime}=\left(E^{\prime}, p^{\prime}, B, \mathscr{A}^{\prime}\right)-$ nочтu главные $G \mid \Lambda$-расслоения, являющиеся композициями накрытия $\nu$ и главных $T$-расслоений $\xi=(E, q, \widetilde{B}, \overline{\mathscr{A}})$ u $\xi^{\prime}=\left(E^{\prime}, q^{\prime}, \widetilde{B}, \overline{\mathscr{A}}^{\prime}\right), \omega u \omega^{\prime}-$ формы $G$-связностей $H$ и $H^{\prime}$ на $E$ и $E^{\prime}$ соответственно $и f: \rho \rightarrow \rho^{\prime}-$ слабый морфизм. Тогда существует такая 1-форма $\widetilde{A}$ на $\widetilde{B}$, что $f^{*} \omega^{\prime}=\omega+q^{*} \widetilde{A}$. При этом $f$ - морфизм почти главных расслоений в том и только том случае,

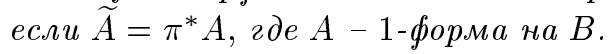


ДоказАТЕльство. Первое утверждение следует из того, что $f$-морфизм главных $T$-расслоений $\xi$ и $\xi^{\prime}$ над $\widetilde{B}$. Предположим, что $f$-морфизм почти главных расслоений $\rho$ и $\rho^{\prime}$. Тогда согласно замечанию после определения 1.7 найдутся такие атласы $\mathscr{A}(\mathscr{U}) \in \mathscr{A}$ и $\mathscr{A}^{\prime}(\mathscr{U}) \in \mathscr{A}^{\prime}$, что $f \circ \psi_{U}=\psi_{U}^{\prime}$ для всех карт $\psi_{U} \in \mathscr{A}(\mathscr{U})$ и $\psi_{U}^{\prime} \in \mathscr{A}^{\prime}(\mathscr{U})$. Формы перехода $\left\{d \psi_{V U}\right\}$ и $\left\{d \psi_{V U}^{\prime}\right\}$ атласов $\mathscr{A}(\mathscr{U})$ и $\mathscr{A}^{\prime}(\mathscr{U})$ совпадают. Поэтому локальные формы $\left\{\omega_{U}\right\}$ и $\left\{\omega_{U}^{\prime}\right\}$ связностей $H$ и $H^{\prime}$ удовлетворяют равенствам $\omega_{V}-\omega_{U}=d \psi_{U V}=\omega_{V}^{\prime}-\omega_{U}^{\prime}$. Таким образом, $\omega_{V}^{\prime}-\omega_{V}=\omega_{U}^{\prime}-\omega_{U}$ и, следовательно, сушествует 1-фформа $A$ на $B$, для которой $\left.A\right|_{U}=\omega_{U}^{\prime}-\omega_{U}$. При этом $f^{*} \omega^{\prime}-\omega=p^{*} A$ и $q^{*} \widetilde{A}=p^{*} A$. Последнее равносильно равенству $\widetilde{A}=\pi^{*} A$.

\section{§3. Групповая структура и характеристические классы}

ОПРЕДЕЛЕНИЕ 3.1. Пусть $\rho=(E, p, B, \mathscr{A})$ и $\rho^{\prime}=\left(E^{\prime}, p^{\prime}, B, \mathscr{A}^{\prime}\right)$ - почти главные $G \mid \Lambda$-расслоения. Рассмотрим атласы $\mathscr{A}(\mathscr{U}) \in \mathscr{A}$ и $\mathscr{A}^{\prime}(\mathscr{U}) \in \mathscr{A}^{\prime}$ и их многозначные функции перехода $\left\{\psi_{V U}^{\alpha}\right\}$ и $\left\{\psi_{V U}^{\prime \alpha}\right\}$. Положим

$$
\psi_{V U}^{\prime \prime \alpha}=\psi_{V U}^{\alpha}+\psi_{V U}^{\prime \alpha}, \quad \rho^{\prime \prime}=\rho+\rho^{\prime} \quad \text { и }\left[\rho^{\prime \prime}\right]=[\rho]+\left[\rho^{\prime}\right]
$$

где $\rho^{\prime \prime}=\left(E^{\prime \prime}, p^{\prime \prime}, B, \mathscr{A}^{\prime \prime}\right)$ - почти главное $G \mid \Lambda$-расслоение с атласом $\mathscr{A}^{\prime \prime}(\mathscr{U}) \in \mathscr{A}^{\prime \prime}$, имеющим многозначные функции перехода $\left\{\psi_{V U}^{\prime \prime \alpha}\right\}$, а $[\rho],\left[\rho^{\prime}\right]$ и $\left[\rho^{\prime \prime}\right]$ - классы эквивалентности соответствующих почти главных расслоений. Формулы (3.1) определяют алгебраическую операцию, превращающую множество $\mathscr{A} \mathscr{P} \mathscr{B}(B \mid \Lambda, G)$ классов эквивалентности почти главных $G \mid \Lambda$-расслоений над $B$ в абелеву группу.

Рассмотрим произведение $E_{0}=\widetilde{B} \times T$, естественную проекцию $q_{0}: E_{0} \rightarrow \widetilde{B}$ и композицию $p_{0}=\pi \circ q_{0}$. Выбрав карту $\nu_{U} \in \tilde{\mathscr{A}}(\mathscr{U})$, положим $\psi_{0 U}(a, \alpha, t)=$ $\left(\nu_{U}(a, \alpha), t\right)$. Этим определены карта $\psi_{0 U}: U \times G \rightarrow \widetilde{B}_{U} \times T$ разложимого расслоения $\psi_{0}=\left(E_{0}, p_{0}, B, G\right)$ и его $\mathscr{A} \mathscr{P} \mathscr{B}$-атлас $\mathscr{A}_{0}(\mathscr{U})=\left\{\psi_{0 U}, U \in \mathscr{U}\right\}$. Если $\mathscr{A}_{0}$ - содержашая построенный атлас $\mathscr{A} \mathscr{P} \mathscr{B}$-структура, то класс эквивалентности $\left[\rho_{0}\right]$ расслоения $\rho_{0}=\left(E_{0}, p_{0}, B, \mathscr{A}_{0}\right)$ является нейтральным элементом группы $\mathscr{A} \mathscr{P} \mathscr{B}(B \mid \Lambda, G)$. Для расслоения $\rho=(E, p, B, \mathscr{A})$ найдется почти главное $G \mid \Lambda$-расслоение $\widehat{\rho}=(\widehat{E}, \widehat{p}, B, \widehat{\mathscr{A}})$ с атласом $\widehat{\mathscr{A}}(\mathscr{U})$, имеюшим многозначные функции перехода $\left\{\widehat{\psi}_{V U}^{\alpha}=\psi_{U V}^{\alpha}\right\}$. При этом $[\widehat{\rho}]=-[\rho]$.

Пусть $H$ и $H^{\prime}-G$-связности на пространствах расслоений $\rho$ и $\rho^{\prime},\left\{\omega_{U}\right\}$ и $\left\{\omega_{U}^{\prime}\right\}-$ их локальные формы связности относительно атласов $\mathscr{A}(\mathscr{U})$ и $\mathscr{A}^{\prime}(\mathscr{U}), \rho^{\prime \prime}=\rho+\rho^{\prime}$ и $\mathscr{A}^{\prime \prime}(\mathscr{U})$ - атлас расслоения $\rho^{\prime \prime}$ из определения 3.1. Тогда сушествует $G$-связность $H^{\prime \prime}$ на $E^{\prime \prime}$ с набором локальных форм связности $\left\{\omega_{U}^{\prime \prime}=\omega_{U}+\omega_{U}^{\prime}\right\}$ относительно $\mathscr{A}^{\prime \prime}(\mathscr{U})$.

Предположим, что $f: \rho \rightarrow \rho^{\prime}$ - морфизм почти главных $G \mid \Lambda$-расслоений $\rho$ и $\rho^{\prime}, H$ и $H^{\prime}-G$-связности на $E$ и $E^{\prime}, \omega$ и $\omega^{\prime}-$ их формы связности, $d \omega=p^{*} \Phi$ и $d \omega^{\prime}=p^{\prime *} \Phi^{\prime}$. Тогда согласно предложению 2.4 существует 1 -форма $A$ на $B$, удовлетворяющая равенству $p^{*} A=f^{*} \omega^{\prime}-\omega$. При этом $d A=\Phi^{\prime}-\Phi$. Следовательно, элемент $[\Phi] \in H^{2}\left(B, \mathbb{R}^{m}\right)$ не зависит от выбора связности $H$ и не меняется при замене $\rho$ изоморфным ему почти главным $G \mid \Lambda$-расслоением.

ОПРЕДЕЛЕНИЕ 3.2. Пусть $\rho=(E, p, B, \mathscr{A})$ - почти главное $G \mid \Lambda$-расслоение и $H-G$-связность на $E$. Когомологический класс $[\Phi] \in H^{2}\left(B, \mathbb{R}^{m}\right)$ проекции на 
базу $B$ формы кривизны связности $H$ мы будем называть характеристическим $I$-классом расслоения $\rho$.

ОПРЕДЕЛЕНИЕ 3.3. Петлю $x \in \Omega_{b b}$ назовем одномернылм $\Lambda$-циклом, если ее гомотопический класс принадлежит подгруппе $\Lambda \subset \Pi=\pi_{1}(B, b)$. Сингулярньй квадрат $c: I^{2} \rightarrow B$ назовем $\Lambda$-квадратом, если $c(0,0)=c(0,1)=c(1,0)=$ $c(1,1)=b$ и каждая его сторона является одномерным $\Lambda$-циклом. Сингулярная цеп, составленная из $\Lambda$-квадратов, будет считаться двумерной $\Lambda$-цепью. Группы $n$-мерных (нормализованных) $\Lambda$-циклов и $\Lambda$-границ обозначим символами $\mathscr{Z}_{n}(B \mid \Lambda)$ и $\mathscr{B}_{n}(B \mid \Lambda), n=1,2$. Положим $\Lambda_{0}=\Lambda /[\Lambda, \Lambda]$ и $H_{n}(B \mid \Lambda)=$ $\mathscr{Z}_{n}(B \mid \Lambda) / \mathscr{B}_{n}(B \mid \Lambda)$.

Отметим, что $H_{1}(B \mid \Lambda)=\Lambda_{0}$ и $H_{2}(B \mid \Lambda)=\pi_{*}\left(H_{2}(\widetilde{B})\right)$.

ОПРЕДЕЛЕНИЕ 3.4. Для замкнутой $\mathbb{R}^{m}$-значной 2-формы $\Phi$ на $B$ определим гомоморфизм $I_{[\Phi]}: H_{2}(B) \rightarrow \mathbb{R}^{m}$ формулой $I_{[\Phi]}([c])=\int_{c} \Phi$. Символом $J_{[\Phi]}$ обозначим его сужение на подгруппу $H_{2}(B \mid \Lambda)$. Для произвольной подгруппы $P \subset \mathbb{R}^{m}$ положим

$$
H^{2}\left(B\left|\Lambda, \mathbb{R}^{m}\right| P\right)=\left\{[\Phi] \in H^{2}\left(B, \mathbb{R}^{m}\right): \operatorname{im} J_{[\Phi]} \subset P\right\}
$$

ПРЕДЛОЖЕНИЕ 3.1. Характеристический I-класс любого почти главного $G \mid \Lambda$-расслоения принадлежит группе $H^{2}\left(B\left|\Lambda, \mathbb{R}^{m}\right| \mathbb{Z}^{k}\right)$. Отображсение $\eta: \mathscr{A} \mathscr{P} \mathscr{B}(B \mid \Lambda, G) \rightarrow H^{2}\left(B\left|\Lambda, \mathbb{R}^{m}\right| \mathbb{Z}^{k}\right)$, ставящее в соответствие классу $[\rho] \in \mathscr{A} \mathscr{P} \mathscr{B}(B \mid \Lambda, G)$ общий характеристический $I$-класс входящих в него почти главных расслоений, является групповым гомоморфизмом.

ДокАЗАТЕльство. По определению $\rho$ является композицией накрытия $\nu$ и главного $T$-расслоения $\xi$ над $\widetilde{B}$. Если $\Phi$ - проекция на $B$ формы кривизны $G$-связности $H$, то $\left[\pi^{*} \Phi\right]$ - характеристический класс расслоения $\xi$. Отсюда и из равенства $H_{2}(B \mid \Lambda)=\pi_{*}\left(H_{2}(\widetilde{B})\right)$ вытекает первое утверждение. Второе утверждение следует из отмеченных в начале параграфа свойств локальных форм связности.

ПРЕДЛОЖЕНИЕ 3.2. Для гомоморфизма $\eta$ из предложения 3.1 существует правый обратный гомоморфизм $\widehat{\eta}: H^{2}\left(B\left|\Lambda, \mathbb{R}^{m}\right| \mathbb{Z}^{k}\right) \rightarrow \mathscr{A} \mathscr{P} \mathscr{B}(B \mid \Lambda, G)$.

ДокАзАтЕльство. Рассмотрим замкнутую 2-форму $\Phi$ на $B$ и предположим, что $[\Phi] \in H^{2}\left(B\left|\Lambda, \mathbb{R}^{m}\right| \mathbb{Z}^{k}\right)$. Для элементов $U \in \mathscr{U}, a \in U$ и $\alpha \in \Delta$ выберем точку $a_{U} \in U, 1$-форму $\omega_{U}$ на $U$, удовлетворяющую равенству $d \omega_{U}=\left.\Phi\right|_{U}$, произвольный путь $x_{U}: I \rightarrow B$ с началом $x_{U}(0)=b$ и конщом $x_{U}(1)=a_{U}$ и такие пути $y_{U}^{a}: I \rightarrow U$ и $x_{U}^{\alpha}: I \rightarrow B$, что $y_{U}^{a}(0)=a_{U}, y_{U}^{a}(1)=a, x_{U}^{\alpha}(0)=b$, $x_{U}^{\alpha}(1)=a_{U}$ и гомотопический класс петли $x_{U}-x_{U}^{\alpha}$ принадлежит $\alpha$. Допустим, что $U, V \in \mathscr{U}$ и $V \cap U \neq \varnothing$. Зафиксируем точку $a_{V U} \in V \cap U$ и положим $y_{U}^{a}=y_{U V}$ и $y_{V}^{a}=y_{V U}$ при $a=a_{V U}$. Символом $\nu_{V U}$ обозначим элемент группы $\Delta$, содержащий гомотопический класс петли $x_{V U}=x_{V}+y_{V U}-y_{U V}-x_{U}$.

Гомотопический класс произвольной петли $x \in \mathscr{B}_{1}(B \mid \Lambda)$ лежит в коммутанте $[\Lambda, \Lambda]$. Поэтому найдутся такие петли $x_{1}, x_{2} \in \mathscr{Z}_{1}(B \mid \Lambda)$, что пути $x_{1}+x_{2}-x_{1}-x_{2}$ и $x$ гомотопны. Рассмотрим связьваюшую их гомотопию $X: I^{2} \rightarrow B$ и положим 
$h_{[\Phi]}(x)=e\left(\int_{X} \Phi\right)$. Если $X^{\prime}-$ другая гомотопия, связывающая те же пути, то $X^{\prime}-X \in \mathscr{Z}_{2}(B \mid \Lambda)$. По условию $e\left(\int_{X^{\prime}-X} \Phi\right)=e\left(I_{[\Phi]}\left(\left[X^{\prime}-X\right]\right)\right)=0$. Это значит, что нами корректно определен гомоморфизм $h_{[\Phi]}: \mathscr{B}_{1}(B \mid \Lambda) \rightarrow T$. Так как $\operatorname{Ext}\left(\Lambda_{0}, T\right)=0$, то гомоморфизм $h_{[\Phi]}$ имеет продолжение $h: \mathscr{Z}_{1}(B \mid \Lambda) \rightarrow T$.

Пусть $\alpha \in \Delta, \beta=\nu_{V U}+\alpha$ и $a \in V \cap U$. Тогда $x_{V U}^{a \alpha}=x_{V}^{\beta}+y_{V}^{a}-y_{U}^{a}-x_{U}^{\alpha}$ - $\Lambda$-цикл. Определим отображение $\psi_{V U}^{\alpha}: V \cap U \rightarrow T$ формулой

$$
\psi_{V U}^{\alpha}(a)=h\left(x_{V U}^{a \alpha}\right)-e\left(\int_{y_{V}^{a}} \omega_{V}\right)+e\left(\int_{y_{U}^{a}} \omega_{U}\right)
$$

Рассмотрим произвольный путь $z: I \rightarrow V \cap U$ с началом $z(0)=a_{V U}$ и концом $z(1)=a$. Положим $x_{V U}^{\alpha}=x_{V U}^{a^{\prime} \alpha}$ при $a^{\prime}=a_{V U}$ и $z_{U}^{a \alpha}=x_{U}^{\alpha}+y_{U}+z-y_{U}^{a}-x_{U}^{\alpha}$. Тогда $x_{V U}^{a \alpha}=-z_{V}^{a \beta}+x_{V U}^{\alpha}+z_{U}^{a \alpha}$ и, следовательно, $h\left(x_{V U}^{a \alpha}\right)=-h\left(z_{V}^{a \beta}\right)+h\left(x_{V U}^{\alpha}\right)+h\left(z_{U}^{a \alpha}\right)$. Выберем гомотопию $Y_{U}^{a \alpha}: I^{2} \rightarrow U$, связьвающую пути $y_{U}^{a}$ и $y_{U}+z$. Определим отображение $X_{U}^{\alpha}: I^{2} \rightarrow B$, полагая $X_{U}^{\alpha}(s, \tau)=x_{U}^{\alpha}(s)$ для всех $s, \tau \in I$. Тогда гомотопия $Z_{U}^{a \alpha}=X_{U}^{\alpha}+Y_{U}^{a \alpha}$ связывает пути $x_{U}^{\alpha}+y_{U}^{a}$ и $x_{U}^{\alpha}+y_{U}+z$. Аналогично построим гомотопию $Z_{V}^{a \beta}=X_{V}^{\beta}+Y_{V}^{a \beta}$, связывающую пути $x_{V}^{\beta}+y_{V}^{a}$ и $x_{V}^{\beta}+y_{V}+z$. Так как $z_{U}^{a \alpha}=\partial Z_{U}^{a \alpha}$ и $z_{V}^{a \beta}=\partial Z_{V}^{a \beta}-\Lambda$-гранищы, то $h\left(z_{U}^{a \alpha}\right)=e\left(\int_{Z_{U}^{a \alpha}} \Phi\right)$ и $h\left(z_{V}^{a \beta}\right)=e\left(\int_{Z_{V}^{a \beta}} \Phi\right)$. По построению интегралы от формы $\Phi$ по $X_{U}^{\alpha}$ и $X_{V}^{\beta}$ равны нулю, а по $Y_{U}^{a \alpha}$ и $Y_{V}^{a \beta}-$ совпадают с интегралами от форм $\omega_{U}$ и $\omega_{V}$ по циклам $y_{U}+z-y_{U}^{a}$ и $y_{V}+z-y_{V}^{a}$ соответственно. Таким образом,

$$
h\left(x_{V U}^{a \alpha}\right)-h\left(x_{V U}^{\alpha}\right)=e\left(\int_{y_{U}+z-y_{U}^{a}} \omega_{U}-\int_{y_{V}+z-y_{V}^{a}} \omega_{V}\right) .
$$

Отсюда и из (3.2) получим:

$$
\psi_{V U}^{\alpha}(a)-\psi_{V U}^{\alpha}\left(a_{V U}\right)=e\left(\int_{z}\left(\omega_{U}-\omega_{V}\right)\right)
$$

Это значит, что отображение $\psi_{V U}^{\alpha}: V \cap U \rightarrow T$ дифференцируемо и

$$
d \psi_{V U}^{\alpha}=d \psi_{V U}=\omega_{U}-\omega_{V}
$$

Если $U, V, W \in \mathscr{U}$ и $W \cap V \cap U \neq \varnothing$, то $\nu_{U W}+\nu_{W V}+\nu_{V U}=0$. Для произвольного $\alpha \in \Delta$ положим $\beta=\nu_{V U}+\alpha$ и $\gamma=\nu_{W V}+\beta$. Тогда $\alpha=\nu_{U W}+\gamma$. При этом согласно $(3.2)\left(\psi_{U W}^{\gamma}+\psi_{W V}^{\beta}+\psi_{V U}^{\alpha}\right)(a)=h\left(x_{U W}^{a \gamma}+x_{W V}^{a \beta}+x_{V U}^{a \alpha}\right)=0$. Отсюда следует сушествование $\Lambda$-разложимого расслоения $\psi=(E, p, B, G)$ с атласом $\mathscr{A}(\mathscr{U})$ и псевдофункциями перехода $\left\{\left(\nu_{V U}, \psi_{V U}^{\alpha}\right)\right\}$. Согласно (3.3) $\mathscr{A}(\mathscr{U})$ - $\mathscr{A} \mathscr{P} \mathscr{B}$-атлас. Символом $\mathscr{A}$ обозначим его класс эквивалентности. Положим 
$\rho=(E, p, B, \mathscr{A})$ и $\widehat{\eta}[\Phi]=[\rho]$. Докажем, что этим корректно определено отображение $\widehat{\eta}: H^{2}\left(B\left|\Lambda, \mathbb{R}^{m}\right| \mathbb{Z}^{k}\right) \rightarrow \mathscr{A} \mathscr{P} \mathscr{B}(B \mid \Lambda, G)$.

Для этого в предыдущих построениях заменим форму $\Phi$ формой $\Phi^{\prime}=\Phi+d A$. Получим почти главное $G$-расслоение $\rho^{\prime}=\left(E^{\prime}, p^{\prime}, B, \mathscr{A}^{\prime}\right)$ с $\mathscr{A} \mathscr{P} \mathscr{B}$-атласом $\mathscr{A}^{\prime}(\mathscr{U}) \in \mathscr{A}^{\prime}$ и многозначными функциями перехода $\left\{\left(\nu_{V U}, \psi_{V U}^{\prime \alpha}\right)\right\}$. Соответствуюший гомоморфизм $h^{\prime}: \mathscr{Z}_{1}(B \mid \Lambda) \rightarrow T$ будет удовлетворять равенству $h^{\prime}(x)=h(x)+e\left(\int_{x} A\right)$. Формы $\omega_{U}$ заменятся формами $\omega_{U}^{\prime}=\omega_{U}+\left.A\right|_{U}$. Поэтому $\psi_{V U}^{\prime \alpha}(a)-\psi_{V U}^{\alpha}(a)=e\left(\int_{x_{V}^{\beta}} A-\int_{x_{U}^{\alpha}} A\right)$. Элементы $\varphi_{U}^{\alpha}=e\left(\int_{x_{U}^{\alpha}} A\right)$ группы $T$ будем считать постоянными отображениями $\varphi_{U}^{\alpha}: U \rightarrow T$. Согласно предыдушему $\psi_{V U}^{\prime \alpha}=\varphi_{V}^{\beta}+\psi_{V U}^{\alpha}-\varphi_{U}^{\alpha}$ для всех $U, V \in \mathscr{U}, \alpha \in \Delta$ и $\beta=\nu_{V U}+\alpha$. Это значит, что почти главные расслоения $\rho$ и $\rho^{\prime}$ изоморфны и, следовательно, отображение $\widehat{\eta}$ определено корректно.

Рассмотрим далее произвольные элементы $[\Phi]$ и $\left[\Phi^{\prime}\right]$ группы $H^{2}\left(B\left|\Lambda, \mathbb{R}^{m}\right| \mathbb{Z}^{k}\right)$ и положим $\left[\Phi^{\prime \prime}\right]=[\Phi]+\left[\Phi^{\prime}\right]$. Как и выше, посредством формулы (3.2) определим отображения $\psi_{V U}^{\alpha}, \psi_{V U}^{\prime \alpha}, \psi_{V U}^{\prime \prime \alpha}: U \cap V \rightarrow T, U, V \in \mathscr{U}, \alpha \in \Delta$, и соответствуюшие им почти главные $G$-расслоения $\rho, \rho^{\prime}$ и $\rho^{\prime \prime}$. Тогда справедливы равенства (3.1), и потому $\widehat{\eta}$ - гомоморфизм.

Из (3.3) вытекает существование $G$-связности $H$ на $E$ с набором локальных форм связности $\left\{\omega_{U}, U \in \mathscr{U}\right\}$. По построению $\Phi$ - проекция на $B$ формы кривизны связности $H$. Таким образом, $\eta[\rho]=[\Phi]$ и, следовательно, $\eta \circ \widehat{\eta}=\mathrm{id}$.

ОПРЕДЕЛЕНИЕ 3.5 . Пусть $\rho=(E, p, B, \mathscr{A})$ - почти главное $G \mid \Lambda$-расслоение с нулевым характеристическим $I$-классом. Тогда существует $G$-связность $H$ на $E$ с формой кривизны $\Omega=0$. Она назьвается плоской связностью. При этом $\rho$ мы будем назьвать плоским $G \mid \Lambda$-расслоением.

ПРЕДЛОЖЕНИЕ 3.3. Пусть $\rho=(E, p, B, \mathscr{A})$ - плоское почти главное $G \mid \Lambda$ расслоение и $H$ - плоская $G$-связность на $E$. Тогда существует гомоморфизм $\tau_{H} \in \operatorname{Hom}\left(\Lambda_{0}, T\right)$, удовлетворяющий равенству $\bar{x}(1)=\bar{x}(0) \cdot \tau_{H}([x])$ для произвольной петли $x \in \mathscr{Z}_{1}(B \mid \Lambda)$ и ее горизонтального лифта $\bar{x}$ относительно $H$.

ДоказатеЛЬСтво. Если $\omega$ - форма связности $H$, то $d \omega=\Omega=0$. Рассмотрим петлю $x \in \mathscr{Z}_{1}(B \mid \Lambda)$ и ее горизонтальный лиф̆т $\bar{x}$ относительно связности $H$. Тогда точки $\bar{x}(0)$ и $\bar{x}(1)$ лежат в одной компоненте связности слоя $p^{-1}(b)$, и потому найдется элемент $\tau_{H}(x) \in T$, удовлетворяющий равенству $\bar{x}(1)=\bar{x}(0) \cdot \tau_{H}(x)$. Выберем другую петлю $y \in \mathscr{Z}_{1}(B \mid \Lambda)$. Если $\bar{y}$ - ее горизонтальный лифт с началом $\bar{y}(0)=\bar{x}(1)$, то $\bar{x}+\bar{y}$-горизонтальный лифт петли $x+y$. Поэтому $\bar{y}(1)=\bar{y}(0) \cdot \tau_{H}(y)$ и $\bar{y}(1)=\bar{x}(0) \cdot \tau_{H}(x+y)$. Отсюда следует, что

$$
\tau_{H}(x+y)=\tau_{H}(x)+\tau_{H}(y) .
$$

$\mathrm{B}$ частности, $\tau_{H}(-x)=-\tau_{H}(x)$. Предположим далее, что циклы $x$ и $y$ гомологичны. Тогда найдутся такие петли $z_{1}, z_{2} \in \mathscr{Z}_{1}(B \mid \Lambda)$, что петля $z=x-y-z_{1}-z_{2}+$ $z_{1}+z_{2}$ гомотопна нулю. Пусть $c: I^{2} \rightarrow B$-кусочно гладкая гомотопия, удовлетворяющая равенствам $c(s, 0)=c(0, r)=c(1, r)=b$ и $c(s, 1)=z(s)$ при всех $s, r \in I$, а 
$\bar{z}$ - горизонтальный лифтт петли $z$. Рассмотрим гомотопию $\bar{c}: I^{2} \rightarrow E$, для которой $p \circ \bar{c}=c, \bar{c}(s, 1)=\bar{z}(s), \bar{c}(0, r)=\bar{z}(0)$ и $\bar{c}(1, r)=\bar{z}(1)$. Положим $\bar{z}_{0}(s)=\bar{c}(s, 0)$ для $s \in I$. Тогда $\partial \bar{c}=\bar{z}-\bar{z}_{0}$ и потому $\int_{\bar{z}-\bar{z}_{0}} \omega=\int_{\partial \bar{c}} \omega=\int_{\bar{c}} d \omega=0$. Так как $\omega\left(\frac{d \bar{z}}{d s}\right) \equiv 0$, то из предыдущего следует равенство $\int_{\bar{z}_{0}} \omega=0$. В силу вертикальности пути $\bar{z}_{0}$ последнее возможно только при $\bar{z}_{0}(1)=\bar{z}_{0}(0)$. Но тогда $\bar{z}(1)=\bar{z}(0)$ и потому $\tau_{H}(z)=0$. Отсюда в силу (3.4) и определения петли $z$ следует равенство $\tau_{H}(x)=\tau_{H}(y)$. Таким образом, формула $\tau_{H}([x])=\tau_{H}(x)$ корректно определяет гомоморфизм $\tau_{H}: \Lambda_{0} \rightarrow T$.

ОПРЕДЕЛЕНИЕ 3.6. Пусть $\rho=(E, p, B, \mathscr{A})$ - почти главное $G \mid \Lambda$-расслоение, $H$ - плоская $G$-связность на $E$ и гомоморфизм $\tau_{H} \in \operatorname{Hom}\left(\Lambda_{0}, T\right)$ удовлетворяет равенству $\bar{x}(1)=\bar{x}(0) \cdot \tau_{H}([x])$ для петель $x \in \mathscr{Z}_{1}(B \mid \Lambda)$ и их горизонтальных лифтов $\bar{x}$ относительно $H$. Тогда $\tau_{H}$ будет называться гомоморфизмом голономии связности $H$.

Рассмотрим гомоморфизм голономии $\tau_{H}: \Lambda_{0} \rightarrow T$ плоской $G$-связности $H$ на пространстве почти главного $G \mid \Lambda$-расслоения $\rho=(E, p, B, \mathscr{A})$ и гомоморфизм $e_{*}: \operatorname{Hom}\left(\Lambda_{0}, \mathbb{R}^{m}\right) \rightarrow \operatorname{Hom}\left(\Lambda_{0}, T\right)$, индуцированный отображением $e: \mathbb{R}^{m} \rightarrow T$. Тогда смежньй класс $\tau_{H}+\mathrm{im} e_{*}$ принадлежит группе $\operatorname{Hom}\left(\Lambda_{0}, T\right) / \operatorname{im} e_{*}=\operatorname{Ext}\left(\Lambda_{0}, \mathbb{Z}^{k}\right)$ и является препятствием для сушествования гомоморфизма $\bar{\tau}_{H}: \Lambda_{0} \rightarrow \mathbb{R}^{m}$, удовлетворяющего равенству $е \circ \bar{\tau}_{H}=\tau_{H}$.

ПрЕДЛОЖЕНИЕ 3.4. Препятствие $\tau_{H}+\operatorname{im} e_{*}$ не зависит от выбора расслоения $\rho$ из класса әквивалентности $[\rho] \in \mathscr{A} \mathscr{P} \mathscr{B}(B \mid \Lambda, G)$ и от выбора плоской $G$-связности $H$ на его тотальном пространстве $E$. Поэтому формула $\varepsilon([\rho])=\tau_{H}+\operatorname{im}_{*}$ корректно определяет отображение $\varepsilon: \operatorname{ker} \eta \rightarrow$ $\operatorname{Ext}\left(\Lambda_{0}, \mathbb{Z}^{k}\right)$.

ДокаЗАтЕльство. Рассмотрим почти главное расслоение $\rho^{\prime}=\left(E^{\prime}, p^{\prime}, B, \mathscr{A}^{\prime}\right)$, плоскую $G$-связность $H^{\prime}$ на его пространстве $E^{\prime}$ и морфизм $f: \rho \rightarrow \rho^{\prime}$. Если $\omega^{\prime}$ - форма связности $H^{\prime}$, то $\omega^{*}=f^{*} \omega^{\prime}$ - форма некоторой $G$-связности $H^{*}$ на $E$. Согласно предложению 2.4 сушествует 1-форма $A$ на $B$, для которой $p^{*} A=\omega^{*}-\omega$. При этом $d A=\Phi^{\prime}-\Phi=0$ и, следовательно, форма $A$ замкнута.

Пусть далее $x \in \mathscr{Z}_{1}(B \mid \Lambda), \bar{x}, \bar{x}^{\prime}$ и $\bar{x}^{*}$ - его горизонтальные лифты относительно связностей $H, H^{\prime}$ и $H^{*}$ соответственно и $\bar{x}(0)=\bar{x}^{*}(0)$. Найдется путь $r: I \rightarrow \mathbb{R}^{m}$, удовлетворяющий равенствам $r(0)=0$ и $\bar{x}^{*}(s)=\bar{x}(s) \cdot e(r(s))$, $s \in I$. Дифференцируя последнее и пользуясь горизонтальностью путей $\bar{x}$ и $\bar{x}^{*}$, получаем: $\frac{d r}{d s}+A\left(\frac{d x}{d s}\right)=0$. Положив $I_{[A]}([x])=-\int_{x} A$, определим гомоморфизм $I_{[A]} \in \operatorname{Hom}\left(\Lambda_{0}, \mathbb{R}^{m}\right)$. Тогда $r(1)=I_{[A]}([x])$. По построению путей $\bar{x}, \bar{x}^{\prime}$ и $\bar{x}^{*}$ и определению гомоморфизмов $\tau_{H}$ и $\tau_{H^{\prime}}$ имеют место равенства: $\bar{x}^{\prime}(s)=f \circ \bar{x}^{*}(s)$ для $s \in I, \bar{x}(1)=\bar{x}(0) \cdot \tau_{H}([x])$ и $\bar{x}^{\prime}(1)=\bar{x}^{\prime}(0) \cdot \tau_{H^{\prime}}([x])$. Согласно доказанному вьше отсюда следует, что $f \circ \bar{x}(0) \cdot \tau_{H^{\prime}}([x])=f \circ \bar{x}(0) \cdot \tau_{H}([x])+e \circ I_{[A]}([x])$. Таким образом, $\tau_{H^{\prime}}-\tau_{H}=e \circ I_{[A]}$ и потому смежные классы $\tau_{H}+\operatorname{im} e_{*}$ и $\tau_{H^{\prime}}+\operatorname{im} e_{*}$ совпадают. 
ОПРЕДЕЛЕНИЕ 3.7 . Пусть $\rho=(E, p, B, \mathscr{A})$ - почти главное $G \mid \Lambda$-расслоение, $H$ - плоская $G$-связность на $E, \tau_{H}: \Lambda_{0} \rightarrow T$ - ее гомоморфизм голономии и $e_{*}: \operatorname{Hom}\left(\Lambda_{0}, \mathbb{R}^{m}\right) \rightarrow \operatorname{Hom}\left(\Lambda_{0}, T\right)$ - гомоморфизм, индуцированный отображением $e: \mathbb{R}^{m} \rightarrow T$. Тогда смежный класс $\tau_{H}+\operatorname{im} e_{*} \in \operatorname{Ext}\left(\Lambda_{0}, \mathbb{Z}^{k}\right)$, являюшийся инвариантом плоского почти главного $G \mid \Lambda$-расслоения $\rho$, мы будем называть его характеристическим є-классом.

ПрЕДЛОЖениЕ 3.5. Отображение $\varepsilon: \operatorname{ker} \eta \rightarrow \operatorname{Ext}\left(\Lambda_{0}, \mathbb{Z}^{k}\right)$, ставящее в соответствие классу эквивалентности плоского почти главного $G \mid \Lambda$-рассло-

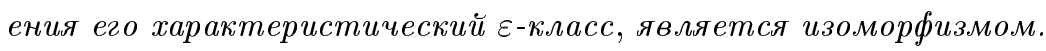

ДокаЗАтЕЛЬСтво. Пусть $\rho=(E, p, B, \mathscr{A})$ - плоское почти главное $G \mid \Lambda$-расслоение, $\mathscr{A}(\mathscr{U})$ - его атлас, ассоциированный с простым покрытием $\mathscr{U}$ базы $B$. Рассмотрим плоскую $G$-связность $H$ на $E$ и ее локальные формы связности $\left\{\omega_{U}\right\}$. Если $\left\{\left(\nu_{V U}, \psi_{V U}^{\alpha}\right)\right\}-$ многозначные функции перехода атласа $\mathscr{A}(\mathscr{U})$, то $\nu_{V U} \equiv$ const. Выберем петлю $x \in \mathscr{Z}_{1}(B \mid \Lambda)$, покроем ее множествами $U_{1}, \ldots, U_{l} \in \mathscr{U}$ и разобьем отрезок $I$ числами $s_{0}=0<s_{1}<\cdots<s_{l}=1$ так, чтобы $x\left(\left[s_{i-1}, s_{i}\right]\right) \subset U_{i}, i=1, \ldots, l$. Положим $x_{i}=\left.x\right|_{\left[s_{i-1}, s_{i}\right]}$. Символом $\bar{x}$ обозначим горизонтальньй лиф̆т петли $x$ относительно $H$. Допустим, что $\bar{x}(0)=\psi_{U_{1}}\left(x(0), \alpha_{1}, t_{1}\right)$. Тогда

$$
\bar{x}\left(s_{i}\right)=\psi_{U_{i+1}}\left(x\left(s_{i}\right), \alpha_{i+1}, t_{i+1}\right),
$$

где $U_{l+1}=U_{1}, \alpha_{i+1}=\nu_{U_{i+1} U_{i}}+\alpha_{i}$ и $t_{i+1}=\psi_{U_{i+1} U_{i}}^{\alpha_{i}}\left(x\left(s_{i}\right)\right)-\int_{x_{i}} \omega_{U_{i}}+t_{i}$, $i=1, \ldots, l$. Таким образом,

$$
\tau_{H}([x])=\sum_{i=1}^{l} \psi_{U_{i+1} U_{i}}^{\alpha_{i}}\left(x\left(s_{i}\right)\right)-\sum_{i=1}^{l} \int_{x_{i}} \omega_{U_{i}} .
$$

Из $(3.5),(3.1)$ и замечания после определения 3.1 следует, что $\tau_{H}([x])+\tau_{H^{\prime}}([x])=$ $\tau_{H^{\prime \prime}}([x])$. Поэтому $\varepsilon-$ гомоморфизм.

Выберем элемент $\lambda \in \operatorname{Ext}\left(\Lambda_{0}, \mathbb{Z}^{k}\right)$ и рассмотрим гомоморфизм $\tau \in \operatorname{Hom}\left(\Lambda_{0}, T\right)$, удовлетворяющий равенству $\lambda=\tau+\operatorname{im} e_{*}$. Точно так, как в предложении 3.2 , для $U, V \in \mathscr{U}, a \in V \cap U$ и $\alpha \in \Delta$ построим элемент $\nu_{V U}$ и петлю $x_{V U}^{a \alpha} \in$ $\mathscr{Z}_{1}(B \mid \Lambda)$. Положим $\psi_{V U}^{\alpha}(a)=\tau\left(\left[x_{V U}^{a \alpha}\right]\right)$. При всех $a, a^{\prime} \in V \cap U$ циклы $x_{V U}^{a \alpha}$ и $x_{V U}^{a^{\prime} \alpha}$ гомологичны. Поэтому $\psi_{V U}^{\alpha} \equiv$ const. Для множеств $W, V, U \in \mathscr{U}$ и элементов $\alpha \in \Delta, \beta=\nu_{V U}+\alpha$ и $\gamma=\nu_{W V}+\beta$ имеем:

$$
\psi_{U W}^{\gamma}+\psi_{W V}^{\beta}+\psi_{V U}^{\alpha} \equiv \tau\left(\left[x_{U W}^{a \gamma}+x_{W V}^{a \beta}+x_{V U}^{a \alpha}\right]\right)=0 .
$$

Отсюда следует существование почти главного $G \mid \Lambda$-расслоения $\rho=(E, p, B, \mathscr{A})$ с атласом $\mathscr{A}(\mathscr{U}) \in \mathscr{A}$ и многозначными функциями перехода $\left\{\left(\nu_{V U}, \psi_{V U}^{\alpha}\right)\right\}$.

В силу тождеств $\psi_{V U}^{\alpha} \equiv$ const на пространстве расслоения $\rho$ определена $G$-связность $H$ с локальными формами связности $\left\{\omega_{U} \equiv 0\right\}$. Рассмотрим горизонтальньй лифт $\bar{x}$ петли $x \in \mathscr{Z}_{1}(B \mid \Lambda)$ относительно $H$. Снова покроем $x$ множествами 
$U_{1}, \ldots, U_{l} \in \mathscr{U}$ и разобьем отрезок $I$ числами $s_{0}=0<s_{1}<\cdots<s_{l}=1$ так, чтобы $x\left(\left[s_{i-1}, s_{i}\right]\right) \subset U_{i}, i=1, \ldots, l$. Тогда согласно (3.5)

$\tau_{H}([x])=\psi_{U_{1} U_{l}}^{\alpha_{l}}+\psi_{U_{l} U_{l-1}}^{\alpha_{l-1}}+\cdots+\psi_{U_{2} U_{1}}^{\alpha_{1}}=\tau\left(\left[x_{U_{1} U_{l}}^{\alpha_{l}}+x_{U_{l} U_{i-1}}^{\alpha_{l-1}}+\cdots+x_{U_{2} U_{1}}^{\alpha_{1}}\right]\right)=\tau([x])$.

Поэтому $\varepsilon([\rho])=\lambda$ и $\varepsilon-$ эпиморфизм.

Предположим, что почти главное $G \mid \Lambda$-расслоение $\rho=(E, p, B, \mathscr{A})$ является композицией накрытия $\nu$ и главного $T$-расслоения $\xi=(E, q, \widetilde{B}, \bar{A}), H$ - плоская $G$-связность на $E$ и гомоморфизм голономии $\tau_{H} \in \operatorname{Hom}\left(\Lambda_{0}, T\right)$ допускает поднятие до гомоморфизма $\tau \in \operatorname{Hom}\left(\Lambda_{0}, \mathbb{R}^{m}\right), \tau_{H}=e \circ \tau$. Рассмотрим продолжение $\bar{\tau} \in \operatorname{Hom}\left(H_{1}(B), \mathbb{R}^{m}\right)$ гомоморфизма $\tau$ и замкнутую 1-форму $A$ на $B$, для которой $I_{[A]}=\bar{\tau}$. Для формы $\omega$ связности $H$ положим $\widehat{\omega}=\omega+p^{*} A$. Тогда $\widehat{\omega}-$ форма некоторой $G$-связности $\widehat{H}$ на $E$.

Предположим, что $x \in \mathscr{Z}_{1}(B \mid \Lambda), \bar{x}$ и $\widehat{x}$ - горизонтальные лиф̆ты петли $x$ относительно $H$ и $\widehat{H}, \bar{x}(0)=\widehat{x}(0)$. Тогда имеется такой путь $r: I \rightarrow \mathbb{R}^{m}$, что $r(0)=0$ и $\widehat{x}(s)=\bar{x}(s) \cdot e(r(s))$ для $s \in I$. При этом

$$
\widehat{\omega}\left(\frac{d \widehat{x}}{d s}\right)=\omega\left(\frac{d \bar{x}}{d s}\right)+\frac{d r}{d s}+A\left(\frac{d x}{d s}\right)
$$

и, следовательно, $\widehat{x}(1)=\bar{x}(1) \cdot e\left(-I_{[A]}([x])\right)$. Отсюда в силу равенств $\bar{x}(1)=$ $\bar{x}(0) \cdot \tau_{H}([x])$ и $\tau_{H}([x])=e\left(I_{[A]}([x])\right)$ вытекает, что $\widehat{x}(1)=\bar{x}(1)$. Таким образом, $\tau_{\widehat{H}}=0 \in \operatorname{Hom}\left(H_{1}(B \mid \Lambda), T\right)$ и $\widehat{H}-T$-связность с тривиальной группой голономии.

Пусть $\rho_{0}=\left(E_{0}, p_{0}, B, \mathscr{A}_{0}\right)$ - почти главное расслоение, класс эквивалентности которого является нейтральньм элементом группы $\mathscr{A} \mathscr{P} \mathscr{B}(B \mid \Lambda, G), H_{0}-$ $G$-связность на $E_{0}, \omega_{0}-$ ее форма связности. Согласно доказанному в предыдушем абзаце сушествует слабый морфизм $f: \rho_{0} \rightarrow \rho$. Так как $d \widehat{\omega}=d \omega_{0}=0$, то найдется замкнутая 1-форма $\widetilde{A}$ на $\widetilde{B}$, удовлетворяюшая равенству $f^{*} \widehat{\omega}=\omega_{0}+q_{0}^{*} \widetilde{A}$.

Рассмотрим произвольную петлю $\widetilde{x}: I \rightarrow \widetilde{B}$, для которой $\widetilde{x}(0)=\widetilde{x}(1)=\widetilde{b}$. Тогда $x=\pi \circ \widetilde{x}-\Lambda$-цикл. Выберем его горизонтальные лифты $\widehat{x}$ и $\bar{x}_{0}$ относительно связностей $\widehat{H}$ и $H_{0}$ так, чтобы $f\left(\bar{x}_{0}(0)\right)=\widehat{x}(0)$. Определим путь $\widehat{x}^{*}: I \rightarrow E$ формулой $\widehat{x}^{*}(s)=f^{-1}(\widehat{x}(s))$. Тогда в силу равенств $\tau_{H_{0}}=\tau_{\widehat{H}}=0$ имеем: $\widehat{x}^{*}(1)=\widehat{x}^{*}(0)$. Поэтому существует такой путь $r^{*}: I \rightarrow \mathbb{R}^{m}$, что $\widehat{x}^{*}(s)=\bar{x}_{0}(s) \cdot e\left(r^{*}(s)\right)$ для $s \in I$ и $r^{*}(0)=r^{*}(1)=0$. При этом $\frac{d r^{*}}{d s}+\widetilde{A}\left(\frac{d \widetilde{x}}{d s}\right)=0$ и $I_{[\widetilde{A}]}([\widetilde{x}])=r^{*}(0)-r^{*}(1)=0$.

Итак, $I_{[\widetilde{A}]}=0 \in \operatorname{Hom}\left(H_{1}(\widetilde{B}), \mathbb{R}^{m}\right)$. Следовательно, найдется гладкое отображение $\mu: \widetilde{B} \rightarrow \mathbb{R}^{m}$, удовлетворяюшее равенству $d \mu=\widetilde{A}$. Для $\alpha \in \Delta$ и $U \in \mathscr{U}$ рассмотрим сечение $s_{U}^{\alpha}: U \rightarrow \widetilde{B}, s_{U}^{\alpha}(a)=\nu_{U}(a, \alpha)$, и положим $\mu_{U}^{\alpha}=\mu \circ s_{U}^{\alpha}$. Для карты $\psi_{0 U} \in \mathscr{A}_{0}(\mathscr{U})$ положим $\psi_{0 U}^{\prime}\left(a, \alpha, t^{\prime}\right)=\psi_{0 U}\left(a, \alpha, t^{\prime}-e \circ \mu_{U}^{\alpha}(a)\right)$. Этим определены карта $\psi_{0 U}^{\prime}: U \times G \rightarrow E_{0 U} \Lambda$-разложимого расслоения $\psi_{0}=\left(E_{0}, p_{0}, B, G\right)$, атлас $\mathscr{A}_{0}^{\prime}(\mathscr{U})=\left\{\psi_{0 U}^{\prime}, U \in \mathscr{U}\right\}$, содержашая его $\mathscr{A} \mathscr{P} \mathscr{B}$-структура $\mathscr{A}_{0}^{\prime}$ и главное $G \mid \Lambda$-расслоение $\rho_{0}^{\prime}=\left(E_{0}, p_{0}, B, \mathscr{A}_{0}^{\prime}\right)$. Поскольку атласы $\mathscr{A}_{0}(\mathscr{U})$ и $\mathscr{A}_{0}^{\prime}(\mathscr{U})$ имеют нулевые функции перехода, то формула $f^{\prime} \circ \psi_{0 U}(a, \alpha, t)=\psi_{0 U}^{\prime}\left(a, \alpha, t+e \circ \mu_{U}^{\alpha}(a)\right)$ определяет морфизм $f^{\prime}: \rho_{0} \rightarrow \rho_{0}^{\prime}$. 
Индуцированные картами $\psi_{0 U}$ и $\psi_{0 U}^{\prime}$ локальные действия групшы $\Delta$ на $E$ удовлетворяют равенству $\left(\delta_{0 U}^{\prime}\right)_{\gamma}=\left(\delta_{0 U}\right)_{\gamma} \cdot e \circ\left(\mu_{U}^{\alpha}-\mu_{U}^{\alpha+\gamma}\right) \circ p_{0}, \alpha, \gamma \in \Delta$. Поэтому форма $\omega_{0}^{\prime}=\omega_{0}+q_{0}^{*} D$ инвариантна относительно $\delta_{0 U}^{\prime}$ и, следовательно, является формой некоторой $G$-связности $H_{0}^{\prime}$ на пространстве расслоения $\rho_{0}^{\prime}$. Так как $f^{*} \widehat{\omega}=\omega_{0}^{\prime}$, то $f: \rho_{0}^{\prime} \rightarrow \rho-$ морфизм. Следовательно, композиция $f \circ f^{\prime}$ является морфизмом расслоений $\rho_{0}$ и $\rho$. Этим доказано, что $\operatorname{ker} \varepsilon=\left[\rho_{0}\right]=0$ в $\mathscr{A} \mathscr{P} \mathscr{B}(B \mid \Lambda, G)$.

Пусть $\imath: \operatorname{ker} \eta \rightarrow \mathscr{A} \mathscr{P} \mathscr{B}(B \mid \Lambda, G)$ - включение, $\zeta=\imath \circ \varepsilon^{-1}$ и

$\operatorname{Hom}\left(H_{2}(B), H_{2}(B \mid \Lambda) ; \mathbb{R}^{m}, \mathbb{Z}^{k}\right)=\left\{h \in \operatorname{Hom}\left(H_{2}(B), \mathbb{R}^{m}\right): h\left(H_{2}(B \mid \Lambda)\right) \subset \mathbb{Z}^{k}\right\}$

Из предложений 3.1-3.5 следует основной результат параграфа.

ТЕОРема 3.6. Последовательность групп и гомоморфизмов

$$
0 \rightarrow \operatorname{Ext}\left(\Lambda_{0}, \mathbb{Z}^{k}\right) \stackrel{\zeta}{\longrightarrow} \mathscr{A} \mathscr{P} \mathscr{B}(B \mid \Lambda, G) \stackrel{\eta}{\longrightarrow} H^{2}\left(B\left|\Lambda, \mathbb{R}^{m}\right| \mathbb{Z}^{k}\right) \rightarrow 0
$$

точна и расщепляется. Поэтому

$$
\begin{aligned}
\mathscr{A} \mathscr{P} \mathscr{B}(B \mid \Lambda, G) & \cong H^{2}\left(B\left|\Lambda, \mathbb{R}^{m}\right| \mathbb{Z}^{k}\right) \oplus \operatorname{Ext}\left(\Lambda_{0}, \mathbb{Z}^{k}\right) \\
& \cong \operatorname{Hom}\left(H_{2}(B), H_{2}(B \mid \Lambda) ; \mathbb{R}^{m}, \mathbb{Z}^{k}\right) \oplus \operatorname{Ext}\left(\Lambda_{0}, \mathbb{Z}^{k}\right) .
\end{aligned}
$$

\section{§4. Главные расслоения со структурной группой $G=\Delta \times T$}

Символом $\mathscr{P} \mathscr{B}(B \mid \Lambda, G)$ обозначим подгруппу групшы $\mathscr{A} \mathscr{P} \mathscr{B}(B \mid \Lambda, G)$, образованную классами эквивалентности главных $G \mid \Lambda$-расслоений над $B$.

ПРЕДЛОЖЕНИЕ 4.1. Пусть $\rho=(E, p, B, \mathscr{A})$ - главное $G \mid \Lambda$-расслоение с характеристическим $I$-классом $[\Phi]$. Тогда существуют регулярные накрытия $\nu=(\widetilde{B}, \pi, B)$ u $\nu^{\prime}=\left(E, \pi^{\prime}, E^{\prime}\right)$ и главнье $T$-расслоения $\xi=(E, q, \widetilde{B}, \bar{A}) u$ $\xi^{\prime}=\left(E^{\prime}, q^{\prime}, B, \mathscr{A}^{\prime}\right)$, обладающие свойствами:

$$
\begin{aligned}
& \pi \circ q=p=q^{\prime} \circ \pi^{\prime} \\
& \pi^{\prime}(v \cdot t)=\pi^{\prime}(v) \cdot t
\end{aligned}
$$

для $v \in E$ и $t \in T ;[\Phi]-$ характеристический I-класс расслоения $\xi^{\prime}$.

ДоказАтЕльство. Выберем атлас $\mathscr{A}(\mathscr{U}) \in \mathscr{A}$. Он обладает функциями перехода $\left\{\left(\nu_{V U}, \psi_{V U}\right)\right\}$. Отсюда следует существование главных расслоений $\nu$ и $\xi$, для которых верна левая часть (4.1). Набор $\left\{\psi_{V U}\right\}$ порождает главное расслоение $\xi^{\prime}$ с атласом $\mathscr{A}^{\prime}(\mathscr{U}) \in \mathscr{A}^{\prime}$, имеющим функции перехода $\left\{\psi_{V U}\right\}$. Для карт $\psi_{U} \in \mathscr{A}(\mathscr{U})$ и $\psi_{U}^{\prime} \in \mathscr{A}^{\prime}(\mathscr{U})$ и элементов $a \in U, \alpha \in \Delta$ и $t \in T$ положим $\pi^{\prime}\left(\psi_{U}(a, \alpha, t)\right)=\psi_{U}^{\prime}(a, t)$. Этим корректно определено гладкое отображение $\pi^{\prime}: E \rightarrow E^{\prime}$, для которого верна правая часть (4.1). Формулы $E_{U}^{\prime}=\left(q^{\prime}\right)^{-1}(U)$ и $\nu_{U}^{\prime}\left(\psi_{U}^{\prime}(a, t), \alpha\right)=\psi_{U}(a, \alpha, t)$ определяют карту $\nu_{U}^{\prime}: E_{U}^{\prime} \times \Delta \rightarrow E_{U}$, а формула $\nu_{V U}^{\prime}\left(\psi_{U}^{\prime}(a, t)\right)=\nu_{V U}(a)-$ функцию перехода $\nu_{V U}^{\prime}: E_{U}^{\prime} \cap E_{V}^{\prime} \rightarrow \Delta$ от карты $\nu_{U}^{\prime}$ к карте $\nu_{V}^{\prime}$. Таким образом, 
$\nu^{\prime}=\left(E, \pi^{\prime}, E^{\prime}\right)$ - регулярное накрытие. Из определения отображения $\pi^{\prime}$ следует (4.2).

Пусть далее $H-G$-связность на пространстве расслоения $\rho, \omega-$ форма связности $H$ и $d \omega=p^{*} \Phi$. Рассмотрим естественную проекцию $j: G \rightarrow T$. В силу (4.2) пара $\left(\pi^{\prime}, j\right)$ является морфизмом главных расслоений $\rho$ и $\xi^{\prime}$ над $B$. Поэтому формула $H_{\pi^{\prime}(v)}^{\prime}=d \pi^{\prime}\left(H_{v}\right), v \in E$, определяет $T$-связность $H^{\prime}$ на $E^{\prime}$. Ее форма связности $\omega^{\prime}$ удовлетворяет равенству $\pi^{\prime *} \omega^{\prime}=\omega$. При этом $p^{*} \Phi=d\left(\pi^{\prime *} \omega^{\prime}\right)=\pi^{\prime *} d \omega^{\prime}$. С дру-

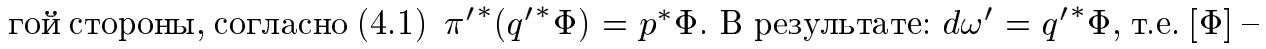
характеристический $I$-класс главного $T$-расслоения $\xi^{\prime}$.

ПРЕДЛОЖЕНИЕ 4.2. Пусть $\xi^{\prime}=\left(E^{\prime}, q^{\prime}, B, \mathscr{A}^{\prime}\right)$ - главное Т-расслоение и $\omega^{\prime}$ - форма некоторой $T$-связности $H^{\prime}$ на $E^{\prime}$. Тогда существуют регулярное накрытие $\nu^{\prime}=\left(E, \pi^{\prime}, E^{\prime}\right)$, главное $G \mid \Lambda$-расслоение $\rho=(E, p, B, \mathscr{A})$ и $G$-связность $H$ на $E$ с формой связности $\omega=\pi^{\prime *} \omega^{\prime}$.

ДокАЗАтельство. Рассмотрим слой $T_{b}=\left(q^{\prime}\right)^{-1}(b)$ и точку $v^{\prime} \in T_{b}$. Тогда точна последовательность $\pi_{1}\left(E^{\prime}, v^{\prime}\right) \stackrel{q_{*}^{\prime}}{\longrightarrow} \pi_{1}(B, b) \stackrel{\partial}{\longrightarrow} \pi_{0}\left(T_{b}, v^{\prime}\right)$, в которой $\pi_{0}\left(T_{b}, v^{\prime}\right)=0$. Поэтому $q_{*}^{\prime}-$ эпиморфизм. Прообраз $\Lambda^{\prime}=\left(q_{*}^{\prime}\right)^{-1}(\Lambda)$ является нормальным делителем группы $\pi_{1}\left(E^{\prime}, v^{\prime}\right)$. Следовательно, существует регулярное накрытие $\pi^{\prime}: E \rightarrow E^{\prime}$, для которого индуцированный гомоморфизм $\pi_{*}^{\prime}: \pi_{1}(E, v) \rightarrow$ $\pi_{1}\left(E^{\prime}, v^{\prime}\right)$ имеет образ $\operatorname{im} \pi_{*}^{\prime}=\Lambda^{\prime}$. При этом $\pi_{1}\left(E^{\prime}, v^{\prime}\right) / \operatorname{im} \pi_{*}^{\prime} \cong \Pi / \Lambda=\Delta$.

Выберем карту $\xi_{U}^{\prime}: U \times T \rightarrow E_{U}^{\prime}$ из атласа $\mathscr{A}^{\prime}(\mathscr{U}) \in \mathscr{A}^{\prime}$, точку $v^{\prime} \in E_{U}^{\prime}$ и стандартньй базис $e_{1}, \ldots, e_{m}$ пространства $\mathbb{R}^{m}$. Гомотопические классы петель $y_{i}^{v^{\prime}}(s)=v^{\prime} \cdot e\left(s e_{i}\right), i=1, \ldots, k$, образуют базис фундаментальной группы $\pi_{1}\left(E_{U}^{\prime}, v^{\prime}\right)$. Так как $\left[y_{i}^{v^{\prime}}\right] \in \operatorname{ker} q_{*}^{\prime}$, то образ индуцированного включением $i_{U}: E_{U}^{\prime} \rightarrow E^{\prime}$ гомоморфизма $\left(i_{U}\right)_{*}: \pi_{1}\left(E_{U}^{\prime}, v^{\prime}\right) \rightarrow \pi_{1}\left(E^{\prime}, v^{\prime}\right)$ лежит в подгруппе $\operatorname{im} \pi_{*}^{\prime}=\Lambda^{\prime} \supset \operatorname{ker} q_{*}^{\prime}$. Поэтому накрытие $\pi^{\prime}$ тривиально над $E_{U}^{\prime}$, т.е. обладает картой $\nu_{U}^{\prime}: E_{U}^{\prime} \times \Delta \rightarrow E_{U}$. Положим $p=q^{\prime} \circ \pi^{\prime}$ и определим отображение $\psi_{U}: U \times G \rightarrow E_{U}$ формулой $\psi_{U}(a, \alpha, t)=\nu_{U}^{\prime}\left(\xi_{U}^{\prime}(a, t), \alpha\right)$. Тогда $\mathscr{U}^{\prime}=\left\{E_{U}^{\prime}, U \in \mathscr{U}\right\}$ - покрытие многообразия $E^{\prime}, \tilde{\mathscr{A}}^{\prime}\left(\mathscr{U}^{\prime}\right)=\left\{\nu_{U}^{\prime}, U \in \mathscr{U}\right\}-$ атлас накрытия $\nu^{\prime}=\left(E, \pi^{\prime}, E^{\prime}\right)$, а $\mathscr{A}(\mathscr{U})=\left\{\psi_{U}, U \in \mathscr{U}\right\}$ - атлас локально тривиального расслоения $(E, p, B, G)$.

Рассмотрим множества $U, V \in \mathscr{U}, V \cap U \neq \varnothing$, и функции перехода $\xi_{V U}^{\prime}$ и $\nu_{V U}^{\prime}$ для карт атласов $\mathscr{A}^{\prime}(\mathscr{U})$ и $\widetilde{\mathscr{A}}^{\prime}\left(\mathscr{U}^{\prime}\right)$. Так как $E_{U}^{\prime} \cap E_{V}^{\prime}-$ связное подмножество многообразия $E^{\prime}$, а группа $\Delta$ дискретна, то $\nu_{V U}^{\prime}: E_{V}^{\prime} \cap E_{U}^{\prime} \rightarrow \Delta$ - постоянное отображение. Его можно отождествить с элементом группы $\Delta$. При этом формула $\psi_{V U}(a)=\left(\nu_{V U}^{\prime}, \xi_{V U}^{\prime}(a)\right)$ определяет функцию перехода $\psi_{V U}: V \cap U \rightarrow G$ от карты $\psi_{U}$ к карте $\psi_{V}$. Следовательно, $\mathscr{A}(\mathscr{U})$ - $\mathscr{P} \mathscr{B}$-атлас, а его класс эквивалентности $\mathscr{A}$ является структурой главного расслоения.

Так как $\pi^{\prime}$ - накрытие, а $\Delta$ - группа его автоморфизмов, то $\omega=\pi^{\prime *} \omega^{\prime}-$ форма некоторой $G$-связности $H$ на $E$.

ПРЕДЛОЖЕНИЕ 4.3. Имеет место включение $\operatorname{ker} \eta \subset \mathscr{P} \mathscr{B}(B \mid \Lambda, G)$.

ДоказАтельство. Рассмотрим элементы $\lambda \in \operatorname{Ext}\left(\Lambda_{0}, \mathbb{Z}^{k}\right)$ и $\tau \in \operatorname{Hom}\left(\Lambda_{0}, T\right)$, для которых $\tau+\operatorname{im} e_{*}=\lambda$. Так как $\operatorname{Ext}\left(H_{1}(B) / \Lambda_{0}, T\right)=0$, то сушествует продолжение $\tau^{\prime} \in \operatorname{Hom}\left(H_{1}(B), T\right)$ гомоморфизма $\tau$. По предложению 3.5 ему соответ- 
ствуют почти главное $G^{\prime} \mid$ П-расслоение $\xi^{\prime}=\left(E^{\prime}, q^{\prime}, B, \mathscr{A}^{\prime}\right)$ и плоская $G^{\prime}$-связность $H^{\prime}$ на $E^{\prime}$ с гомоморфизмом голономии $\tau_{H^{\prime}}=\tau^{\prime}$. При этом $\Delta^{\prime}=\Pi / \Lambda^{\prime}=0$, $G^{\prime}=T$ и, следовательно, $\xi^{\prime}$ - главное $T$-расслоение. Предположим, что $\omega^{\prime}$ - форма связности $H^{\prime}$. Тогда согласно предложению 4.2 найдутся главное $G$-расслоение $\rho=(E, p, B, \mathscr{A})$, регулярное накрытие $\pi^{\prime}: E \rightarrow E^{\prime}$ и $G$-связность $H$ на $E$ с формой связности $\omega=\pi^{\prime *} \omega^{\prime}$. Так как $d \omega=\pi^{\prime *} d \omega^{\prime}=0$, то $H$ - плоская связность. Для любого элемента $[x] \in \Lambda_{0}$ верно равенство $\tau_{H}([x])=\tau_{H^{\prime}}([x])=\tau([x])$. Следовательно, $\varepsilon([\rho])=\lambda$. В силу предложения 3.5 этим утверждение доказано.

ПРЕДЛОЖЕНИЕ 4.4. Почти главное $G \mid \Lambda$-расслоение $\rho$ с базой $B$ и характеристическим I-классом $[\Phi]$ является главным расслоением со структурной группой $G$ тогда и только тогда, когда $I_{[\Phi]} \in \operatorname{Hom}\left(H_{2}(B), \mathbb{Z}^{k}\right)$.

ДокАЗАтЕльство. Если $\rho$ - главное $G$-расслоение, то по предложению 4.1 существует главное $T$-расслоение с тем же характеристическим $I$-классом $[\Phi]$. Согласно предложению $3.1 \mathrm{im} I_{[\Phi]} \subset \mathbb{Z}^{k}$. Допустим далее, что $I_{[\Phi]} \in \operatorname{Hom}\left(H_{2}(B), \mathbb{Z}^{k}\right)$. Тогда по предложению 3.2 найдется главное $T$-расслоение $\xi^{\prime}=\left(E^{\prime}, q^{\prime}, B, \mathscr{A}^{\prime}\right)$ с характеристическим $I$-классом $[\Phi]$. Согласно предложению 4.2 сушествует главное $G \mid \Lambda$-расслоение $\widehat{\rho}$ с базой $B$ и тем же характеристическим $I$-классом. При этом $[\rho]-[\widehat{\rho}] \in \operatorname{ker} \eta$ и $[\widehat{\rho}] \in \mathscr{P} \mathscr{B}(B \mid \Lambda, G)$. Отсюда и из предложения 4.3 следует, что $[\rho] \in \mathscr{P} \mathscr{B}(B \mid \Lambda, G)$, т.е. $\rho$ - главное расслоение со структурной группой $G$.

Рассмотрим изоморфизм $h: H^{2}\left(B, \mathbb{R}^{m}\right) \rightarrow \operatorname{Hom}\left(H_{2}(B), \mathbb{R}^{m}\right)$, определенньй формулой $h([\Phi])=I_{[\Phi]}$. Положим $\eta_{0}=h \circ \eta \mid \mathscr{P} \mathscr{B}(B \mid \Lambda, G)$. Из теоремы 3.6 и предложения 4.4 следует

ТЕОРема 4.5. Последовательность

$$
0 \rightarrow \operatorname{Ext}\left(\Lambda_{0}, \mathbb{Z}^{k}\right) \stackrel{\zeta}{\longrightarrow} \mathscr{P} \mathscr{B}(B \mid \Lambda, G) \stackrel{\eta_{0}}{\longrightarrow} \operatorname{Hom}\left(H_{2}(B), \mathbb{Z}^{k}\right) \rightarrow 0
$$

точна и расщепляется. Поэтому

$$
\mathscr{P} \mathscr{B}(B \mid \Lambda, G) \cong \operatorname{Hom}\left(H_{2}(B), \mathbb{Z}^{k}\right) \oplus \operatorname{Ext}\left(\Lambda_{0}, \mathbb{Z}^{k}\right)
$$

\section{§5. Классификация относительно слабых морфизмов}

Рассмотрим подгруппу $H^{2}\left(B\left|\Lambda, \mathbb{R}^{m}\right| 0\right) \subset H^{2}\left(B\left|\Lambda, \mathbb{R}^{m}\right| \mathbb{Z}^{k}\right)$ и соответствующую факторгруппу $H_{0}^{2}\left(B\left|\Lambda, \mathbb{R}^{m}\right| \mathbb{Z}^{k}\right)=H^{2}\left(B\left|\Lambda, \mathbb{R}^{m}\right| \mathbb{Z}^{k}\right) / H^{2}\left(B\left|\Lambda, \mathbb{R}^{m}\right| 0\right)$. Для смежных классов $[[\Phi]]=[\Phi]+H^{2}\left(B\left|\Lambda, \mathbb{R}^{m}\right| 0\right)$ положим $h_{1}([[\Phi]])=J_{[\Phi]}$. Этим определен изоморфизм $h_{1}: H_{0}^{2}\left(B\left|\Lambda, \mathbb{R}^{m}\right| \mathbb{Z}^{k}\right) \rightarrow \operatorname{Hom}\left(H_{2}(B \mid \Lambda), \mathbb{Z}^{k}\right)$.

Обозначим символом $\mathscr{A} \mathscr{P} \mathscr{B}_{1}(B \mid \Lambda, G)$ множество классов эквивалентности почти главных $G \mid \Lambda$-расслоений над $B$ относительно слабых морфизмов. Для классов $[\rho] \in \mathscr{A} \mathscr{P} \mathscr{B}(B \mid \Lambda, G)$ и $[\rho]_{1} \in \mathscr{A} \mathscr{P} \mathscr{B}_{1}(B \mid \Lambda, G)$ имеет место включение $[\rho] \subset[\rho]_{1}$. Поэтому мы будем полагать $[\rho]_{1}=[[\rho]]$.

ПРЕДЛОЖЕНИЕ 5.1. Если $\left[\rho_{0}\right]$ является нейтральным әлементом группь $\mathscr{A} \mathscr{P} \mathscr{B}(B \mid \Lambda, G)$, mo $\left[\left[\rho_{0}\right]\right]$ - eе nодгруппа u

$$
\mathscr{A} \mathscr{P} \mathscr{B}(B \mid \Lambda, G) /\left[\left[\rho_{0}\right]\right]=\mathscr{A} \mathscr{P} \mathscr{B}_{1}(B \mid \Lambda, G) .
$$


ДокАЗАтЕльство. Выберем почти главные расслоения $\rho_{0}^{\prime}$ и $\rho_{0}^{\prime \prime}$, для которых $\left[\rho_{0}^{\prime}\right]$ и $\left[\rho_{0}^{\prime \prime}\right]$ принадлежат слабому классу $\left[\left[\rho_{0}\right]\right]$. По построению расслоения $\rho_{0}$ их $\mathscr{A} \mathscr{P} \mathscr{B}$-структуры содержат атласы с многозначными функциями перехода $\left\{\varphi_{V}^{\prime \beta}-\varphi_{U}^{\prime \alpha}\right\}$ и $\left\{\varphi_{V}^{\prime \prime}{ }_{V}^{\beta}-\varphi_{U}^{\prime \prime \alpha}\right\}$, где $\beta=\nu_{V U}+\alpha$ и $\varphi_{U}^{\prime \alpha}, \varphi_{U}^{\prime \prime \alpha}: U \rightarrow T$ - гладкие отображения. Если $\bar{\rho}_{0}$ - почти главное $G \mid \Lambda$-расслоение и некоторьй его $\mathscr{A} \mathscr{P} \mathscr{B}$-атлас имеет многозначные функции перехода $\left\{\varphi_{V}^{\prime \beta}-\varphi_{V}^{\prime \prime \beta}-\varphi_{U}^{\prime \alpha}+\varphi_{U}^{\prime \prime \alpha}\right\}$, то $\left[\rho_{0}^{\prime}\right]-\left[\rho_{0}^{\prime \prime}\right]=\left[\bar{\rho}_{0}\right] \in\left[\left[\rho_{0}\right]\right]$. Этим доказано, что $\left[\left[\rho_{0}\right]\right]-$ подгруппа группы $\mathscr{A} \mathscr{P} \mathscr{B}(B \mid \Lambda, G)$.

Рассмотрим далее почти главное $G \mid \Lambda$-расслоение $\rho=(E, p, B, \mathscr{A})$ с атласом $\mathscr{A}(\mathscr{U}) \in \mathscr{A}$ и многозначньми функциями перехода $\left\{\psi_{V U}^{\alpha}\right\}$. Положим

$$
\psi_{V U}^{\prime \alpha}=\varphi_{V}^{\prime \beta}+\psi_{V U}^{\alpha}-\varphi_{U}^{\prime \alpha}
$$

Тогда определено почти главное расслоение $\rho^{\prime}$ с $\mathscr{A} \mathscr{P} \mathscr{B}$-атласом, имеющим многозначные функции перехода $\left\{\psi_{V U}^{\prime \alpha}\right\}$. Согласно (5.1) оно слабо изоморфно расслоению $\rho$ и $[\rho]+\left[\rho_{0}^{\prime}\right]=\left[\rho^{\prime}\right]$. Поэтому $[\rho]+\left[\left[\rho_{0}\right]\right] \subset[[\rho]]$.

$\mathrm{C}$ другой стороны, если почти главные $G \mid \Lambda$-расслоения $\rho$ и $\rho^{\prime}$ слабо изоморфны, то их $\mathscr{A} \mathscr{P} \mathscr{B}$-структуры обладают атласами с многозначньми функциями перехода $\left\{\psi_{V U}^{\alpha}\right\}$ и $\left\{\psi_{V U}^{\prime \alpha}\right\}$, удовлетворяющими равенству (5.1). Так как $\left\{\varphi_{V}^{\prime \beta}-\varphi_{U}^{\prime \alpha}\right\}-$ многозначные функции перехода $\mathscr{A} \mathscr{P} \mathscr{B}$-атласа расслоения $\rho_{0}^{\prime}$, то отсюда следует, что $\left[\rho^{\prime}\right]=[\rho]+\left[\rho_{0}^{\prime}\right]$. Таким образом, $[[\rho]] \subset[\rho]+\left[\left[\rho_{0}\right]\right]$ и предложение доказано.

Согласно предложению 5.1 множество $\mathscr{A} \mathscr{P} \mathscr{B}_{1}(B \mid \Lambda, G)$ является группой. В явном виде соответствующая алгебраическая операция может быть определена формулой $[[\rho]]+\left[\left[\rho^{\prime}\right]\right]=\left[[\rho]+\left[\rho^{\prime}\right]\right]$.

Пусть $\rho=(E, p, B, \mathscr{A})$ и $\rho^{\prime}=\left(E^{\prime}, p^{\prime}, B, \mathscr{A}^{\prime}\right)$ - почти главные $G \mid \Lambda$-расслоения с характеристическими $I$-классами $[\Phi]$ и $\left[\Phi^{\prime}\right]$. Это значит, что на $E$ и $E^{\prime}$ имеются $G$-связности с формами кривизны $p^{*} \Phi$ и $p^{\prime *} \Phi^{\prime}$. Рассмотрим накрытие $\nu$ и главные $T$-расслоения $\xi=(E, q, \widetilde{B}, \overline{\mathscr{A}})$ и $\xi^{\prime}=\left(E^{\prime}, q^{\prime}, \widetilde{B}, \overline{\mathscr{A}}^{\prime}\right)$, для которых $p=\pi \circ q$ и $p^{\prime}=\pi \circ q^{\prime}$. Тогда $\left[\pi^{*} \Phi\right]$ и $\left[\pi^{*} \Phi^{\prime}\right]-$ характеристические $I$-классы расслоений $\xi$ и $\xi^{\prime}$. Если почти главные расслоения $\rho$ и $\rho^{\prime}$ слабо изоморфны, то изоморфны главные расслоения $\xi$ и $\xi^{\prime}$ и потому $\left[\pi^{*} \Phi\right]=\left[\pi^{*} \Phi^{\prime}\right]$. Последнее эквивалентно равенству $[[\Phi]]=\left[\left[\Phi^{\prime}\right]\right]$. Таким образом, смежный класс $[[\Phi]]=[\Phi]+H^{2}\left(B\left|\Lambda, \mathbb{R}^{m}\right| 0\right)$ является инвариантом расслоения $\rho$ относительно слабых морфизмов. То же самое справедливо для соответствуюшего ему гомоморфизма $J_{[\Phi]} \in \operatorname{Hom}\left(H_{2}(B \mid \Lambda), \mathbb{Z}^{k}\right)$.

ОПРЕДЕЛЕНИЕ 5.1. Пусть $\rho=(E, p, B, \mathscr{A})$ - почти главное $G \mid \Lambda$-расслоение, $\Phi$ - проекция на базу $B$ формы кривизны некоторой $G$-связности $H$ на $E$ и $[\Phi] \in H^{2}\left(B\left|\Lambda, \mathbb{R}^{m}\right| \mathbb{Z}^{k}\right)$ - когомологический класс формы $\Phi$. Тогда смежный класс $[[\Phi]]=[\Phi]+H^{2}\left(B\left|\Lambda, \mathbb{R}^{m}\right| 0\right)$ будет называться характеристическим $J$-классом расслоения $\rho$, а определенньй формулой $J_{[\Phi]}([c])=\int_{c} \Phi$ гомоморфизм $J_{[\Phi]} \in \operatorname{Hom}\left(H_{2}(B \mid \Lambda), \mathbb{Z}^{k}\right)$ - его характеристическим гомоморфизмом.

Tеорема 5.2. Отображсения $\eta_{1}: \mathscr{A} \mathscr{P} \mathscr{B}_{1}(B \mid \Lambda, G) \rightarrow H_{0}^{2}\left(B\left|\Lambda, \mathbb{R}^{m}\right| \mathbb{Z}^{k}\right)$ u $\varkappa_{1}=h_{1} \circ \eta_{1}: \mathscr{A} \mathscr{P} \mathscr{B}_{1}(B \mid \Lambda, G) \rightarrow \operatorname{Hom}\left(H_{2}(B \mid \Lambda), \mathbb{Z}^{k}\right)$, cтавящие в соответствие слабому классу эквивалентности $[[\rho]]$ почти главного $G \mid \Lambda$-расслое- 
ния $\rho$ его характеристический J-класс [[Ф]] и характеристический гомоморфизм $J_{[\Phi]}$, являются изоморфизмами.

ДоказАТЕльство. Так как $\eta_{1}([[\rho]])=[\eta([\rho])]$, то $\eta_{1}-$ гомоморфизм. Из сюръективности $\eta$ следует, что $\eta_{1}$ - эпиморфизм. Рассмотрим почти главные $G \mid \Lambda$-расслоения $\rho$ и $\rho_{0}$ с характеристическими $I$-классами $[\Phi]$ и $\left[\Phi_{0}\right]=0$. Предположим, что $[[\Phi]]=0$. Если $\rho$ и $\rho_{0}-$ композиции накрытия $\nu$ и главных $T$-расслоений $\xi$ и $\xi_{0}$, то $\left[\pi^{*} \Phi\right]=\left[\pi^{*} \Phi_{0}\right]=0$. Так как $H_{1}(\widetilde{B})=0$, то отсюда следует сушествование морфизма $f: \xi \rightarrow \xi_{0}$. При этом $f: \rho \rightarrow \rho_{0}$ - слабый морфизм почти главных расслоений. Таким образом, $[[\rho]]=\left[\left[\rho_{0}\right]\right]=0$ в $\mathscr{A} \mathscr{P} \mathscr{B}_{1}(B \mid \Lambda, G)$ и $\eta_{1}$ - мономорфизм. Из изоморфности $\eta_{1}$ следует изоморфность отображения $\varkappa_{1}$.

ПРЕДЛОЖЕНИЕ 5.3. Почти главное $G \mid \Lambda$-расслоение $\rho$ с базой $B$ слабо изоморфно некоторому главному $G \mid \Lambda$-расслоению над $B$ тогда и только тогда, когда его характеристический гомоморфизм $J_{[\Phi]} \in \operatorname{Hom}\left(H_{2}(B \mid \Lambda), \mathbb{Z}^{k}\right)$ может бъть продолжен до гомоморфизма из группьи $\operatorname{Hom}\left(H_{2}(B), \mathbb{Z}^{k}\right)$.

ДокАЗАТЕльство. Если $\rho^{\prime}-$ главное $G \mid \Lambda$-расслоение с характеристическим $I$-классом $\left[\Phi^{\prime}\right]$ и $[[\rho]]=\left[\left[\rho^{\prime}\right]\right]$, то по предложению $4.4 I_{\left[\Phi^{\prime}\right]} \in \operatorname{Hom}\left(H_{2}(B), \mathbb{Z}^{k}\right)$, а по теореме $5.2 J_{[\Phi]}=J_{\left[\Phi^{\prime}\right]}$. При этом $I_{\left[\Phi^{\prime}\right]}-$ продолжение гомоморфизма $J_{\left[\Phi^{\prime}\right]}$. Допустим далее, что гомоморфизм $J_{[\Phi]}$ имеет продолжение $\lambda \in \operatorname{Hom}\left(H_{2}(B), \mathbb{Z}^{k}\right)$. Тогда согласно теореме 4.5 найдется главное $G \mid \Lambda$-расслоение $\rho^{\prime}$ с характеристическим $I$-классом $\left[\Phi^{\prime}\right]$, для которого $I_{\left[\Phi^{\prime}\right]}=\lambda$. По построению $J_{\left[\Phi^{\prime}\right]}=J_{[\Phi]}$, откуда в силу той же теоремы 5.2 следует равенство $\left[\left[\rho^{\prime}\right]\right]=[[\rho]]$.

Подгруппу слабых классов $[[\rho]] \in \mathscr{A} \mathscr{P} \mathscr{B}_{1}(B \mid \Lambda, G)$, содержащих главные $G \mid \Lambda$-расслоения, обозначим символом $\mathscr{P} \mathscr{B}_{1}(B \mid \Lambda, G)$.

TЕOPEMA 5.4. Пусть гомоморфизмы

$$
\begin{aligned}
& i_{*}: \operatorname{Hom}\left(H_{2}(B), \mathbb{Z}^{k}\right) \rightarrow \operatorname{Hom}\left(H_{2}(B \mid \Lambda), \mathbb{Z}^{k}\right), \\
& j_{*}: \operatorname{Ext}\left(H_{2}(B) / H_{2}(B \mid \Lambda), \mathbb{Z}^{k}\right) \rightarrow \operatorname{Ext}\left(H_{2}(B), \mathbb{Z}^{k}\right)
\end{aligned}
$$

индуцировань короткой точной последовательностью

$$
0 \rightarrow H_{2}(B \mid \Lambda) \stackrel{i}{\rightarrow} H_{2}(B) \stackrel{j}{\rightarrow} H_{2}(B) / H_{2}(B \mid \Lambda) \rightarrow 0
$$

Тогда $\mathscr{P} \mathscr{B}_{1}(B \mid \Lambda, G) \cong \operatorname{im} i_{*} u \mathscr{A} \mathscr{P} \mathscr{B}_{1}(B \mid \Lambda, G) / \mathscr{P} \mathscr{B}_{1}(B \mid \Lambda, G) \cong \operatorname{ker} j_{*}$.

ДокАЗАТЕЛЬСтво. Первый изоморфизм следует из теоремы 5.2 и предложения 5.3, а для вывода второго нужно воспользоваться точностью последовательности

$$
\stackrel{i_{*}}{\longrightarrow} \operatorname{Hom}\left(H_{2}(B \mid \Lambda), \mathbb{Z}^{k}\right) \longrightarrow \operatorname{Ext}\left(H_{2}(B) / H_{2}(B \mid \Lambda), \mathbb{Z}^{k}\right) \stackrel{j_{*}}{\longrightarrow}
$$

СлеДСТВИЕ. Если группа $H_{2}(B) / H_{2}(B \mid \Lambda)$ конечно порождена, то факторгруппа $\mathscr{A} \mathscr{P} \mathscr{B}_{1}(B \mid \Lambda, G) / \mathscr{P} \mathscr{B}_{1}(B \mid \Lambda, G)$ конечна. 


\section{§6. Примеры}

6.1. Пусть $B=\mathbb{R} P^{2}$. Тогда $\Pi \cong \mathbb{Z}_{2}$ и $H^{2}(B, \mathbb{R})=0$. При этом $H^{2}(B|\Lambda, \mathbb{R}| \mathbb{Z})$ $=0$ для $\Lambda \subset \Pi$. Если $\Delta=\Pi / \Lambda$ и $G=\Delta \times T^{1}$, то по теореме 5.2 любое почти главное $G \mid \Lambda$-расслоение $\rho=(E, p, B, \mathscr{A})$ удовлетворяет равенству $[[\rho]]=0$. В частности, $E \approx S^{2} \times T^{1}$ при $\Lambda=0$ и $E \approx \mathbb{R} P^{2} \times T^{1}$ при $\Lambda=$ П. Отсюда следует, что композиция расслоения Хопфа $S^{3} \rightarrow S^{2}$ и стандартного накрытия $S^{2} \rightarrow \mathbb{R} P^{2}$ не допускает структуры почти главного и, тем более, главного $G \mid \Lambda$-расслоения.

6.2. Предположим, что $f_{1}$ и $f_{2}$-нормированные формы объема на $S^{2}$ и $T^{2}$ соот-

ветственно. При этом $\int_{S^{2}} f_{1}=\int_{T^{2}} f_{2}=1$. Пользуясь проекциями $p_{1}: S^{2} \times T^{2} \rightarrow S^{2}$ и $p_{2}: S^{2} \times T^{2} \rightarrow T^{2}$, построим форму $\Phi=p_{1}^{*} f_{1}+\sqrt{2} p_{2}^{*} f_{2}$ на $B=S^{2} \times T^{2}$. Тогда $[\Phi] \in H^{2}(B|\Lambda, \mathbb{R}| \mathbb{Z})$ для $\Lambda=0$ и по предложению 3.2 сушествует почти главное $G \mid \Lambda$-расслоение $\rho=(E, p, B, \mathscr{A})$ с характеристическим $I$-классом $[\Phi]$. Так как $I_{[\Phi]} \notin \operatorname{Hom}\left(H_{2}(B), \mathbb{Z}\right)$, то согласно предложению $4.4 \rho$ не может быть главным $G \mid \Lambda$-расслоением. Однако гомоморфизм $J_{[\Phi]} \in \operatorname{Hom}\left(H_{2}(B \mid \Lambda), \mathbb{Z}\right)$ имеет продолжение $I_{\left[\Phi^{\prime}\right]} \in \operatorname{Hom}\left(H_{2}(B), \mathbb{Z}\right)$, где $\Phi^{\prime}=p_{1}^{*} f_{1}+p_{2}^{*} f_{2}$. Поэтому $\rho$ слабо изоморфно главному $G \mid \Lambda$-расслоению с характеристическим $I$-классом $\left[\Phi^{\prime}\right]$.

6.3. Стандартное клеточное разбиение бутылки Клейна $\mathbb{K}^{2}$ и окружности $S^{1}$ индуцирует клеточное разбиение связной суммы $B=\left(\mathbb{K}^{2} \times S^{1}\right) \#\left(\mathbb{K}^{2} \times S^{1}\right)$, состоящее из одной нульмерной, шести одномерных, семи двумерных и двух трехмерных клеток. При этом

$$
\begin{gathered}
\Pi=\pi_{1}(B)=\left\langle\alpha, \beta, \gamma, \alpha^{\prime}, \beta^{\prime}, \gamma^{\prime} \|[\alpha, \gamma],[\beta, \gamma],\left[\alpha^{\prime}, \gamma^{\prime}\right],\left[\beta^{\prime}, \gamma^{\prime}\right],\right. \\
\left.\alpha+\beta+\alpha-\beta, \alpha^{\prime}+\beta^{\prime}+\alpha^{\prime}-\beta^{\prime}\right\rangle, \\
H_{2}(B)=\langle P\rangle \oplus\langle Q\rangle \oplus\left\langle Q^{\prime}\right\rangle \oplus\left\langle P-P^{\prime} \| 2\left(P-P^{\prime}\right)\right\rangle \cong \mathbb{Z} \oplus \mathbb{Z} \oplus \mathbb{Z} \oplus \mathbb{Z}_{2} .
\end{gathered}
$$

Рассмотрим подгруппу $\Lambda \subset$ П, порожденную элементами $2 \alpha, \alpha+\beta, \gamma, \alpha-\alpha^{\prime}$, $2 \alpha^{\prime}, \alpha^{\prime}+\beta^{\prime}, \gamma^{\prime}$. Тогда $\Delta=\Pi / \Lambda=\langle\alpha+\Lambda \| 2(\alpha+\Lambda)\rangle \cong \mathbb{Z}_{2}$. Соответствующая подгруппа $H_{2}(B \mid \Lambda) \subset H_{2}(B)$ имеет набор образующих $2 P, P+Q, P-P^{\prime}, P^{\prime}+Q^{\prime}$. Поэтому

$$
H_{2}(B) / H_{2}(B \mid \Lambda)=\left\langle P+H_{2}(B \mid \Lambda) \| 2\left(P+H_{2}(B \mid \Lambda)\right)\right\rangle \cong \mathbb{Z}_{2} .
$$

Последовательность (5.2) имеет вид

$$
0 \rightarrow 2 \mathbb{Z} \oplus \mathbb{Z} \oplus \mathbb{Z} \oplus \mathbb{Z}_{2} \stackrel{i}{\rightarrow} \mathbb{Z} \oplus \mathbb{Z} \oplus \mathbb{Z} \oplus \mathbb{Z}_{2} \stackrel{j}{\rightarrow} \mathbb{Z}_{2} \rightarrow 0
$$

Следовательно, $\operatorname{Ext}\left(H_{2}(B), \mathbb{Z}\right) \cong \mathbb{Z}_{2}$ и $\operatorname{Ext}\left(H_{2}(B) / H_{2}(B \mid \Lambda), \mathbb{Z}\right) \cong \mathbb{Z}_{2}$, но гомоморфизм $j_{*}: \mathbb{Z}_{2} \rightarrow \mathbb{Z}_{2}$ тривиален. Согласно теореме 5.4 это означает, что для $G=\Delta \times T^{1}$ имеет место равенство $\mathscr{A} \mathscr{P} \mathscr{B}_{1}(B \mid \Lambda, G) / \mathscr{P} \mathscr{B}_{1}(B \mid \Lambda, G) \cong \mathbb{Z}_{2}$. Определим гомоморфизм $l \in \operatorname{Hom}\left(H_{2}(B), \mathbb{R}\right)$, положив $l(P)=l\left(P^{\prime}\right)=1 / 2$ и $l(Q)=l\left(Q^{\prime}\right)=-1 / 2$. Тогда $l(2 P)=1$ и $l(P+Q)=l\left(P^{\prime}+Q^{\prime}\right)=l\left(P-P^{\prime}\right)=0$, т.е. $l_{\Lambda}=\left.l\right|_{H_{2}(B \mid \Lambda)} \in \operatorname{Hom}\left(H_{2}(B \mid \Lambda), \mathbb{Z}\right)$. Поэтому сушествует когомологический класс $[\Phi] \in H^{2}(B|\Lambda, \mathbb{R}| \mathbb{Z})$, удовлетворяющий равенству $I_{[\Phi]}=l$. По предложению 3.2 ему соответствует почти главное $G \mid \Lambda$-расслоение $\rho=(E, p, B, \mathscr{A})$ с характеристическим $I$-классом $[\Phi]$. Поскольку гомоморфизм $J_{[\Phi]}=l_{\Lambda}$ не имеет продолжения до гомоморфизма из $\operatorname{Hom}\left(H_{2}(B), \mathbb{Z}\right)$, то согласно предложению 5.3 $\rho$ не может быть даже слабо изоморфно главному $G \mid \Lambda$-расслоению над $B$. 


\section{§7. Приложения}

На гладком многообразии $B$ рассмотрим риманову или лоренцеву метрику $h$, замкнутую 2-форму $F$ и гладкую функцию $u$. Четверка $\Gamma=(B, h, F, u)$ называется гироскопической системой. В механике $B$ играет роль конфигурационного многообразия системы, $h / 2$ - формы кинетической энергии, $F$ - формы гироскопических сил, а $u$ - потенциальной энергии. В общей теории относительности $B$ - пространственно-временное многообразие, $h$ - гравитационный потенциал, $F-$ форма электромагнитного поля, $u \equiv$ const.

Выберем точки $a, b \in B$, положительное число $\delta \in \mathbb{R}$ и обозначим буквой $M_{\delta}$ бесконечномерное многообразие кусочно гладких путей $x:[0, \delta] \rightarrow B$ с началом $x(0)=a$ и концом $x(\delta)=b$. Касательное пространство $T_{x} M_{\delta}$ состоит из векторных полей вдоль $x$, обращающихся в нуль в точках $a$ и $b$. Пусть $\nabla$ - оператор ковариантного дифференцирования в $(B, h)$, а $F^{\#}$ - линейный оператор, определенньй формулой $h\left(F^{\#}(X), Y\right)=F(X, Y)$. Для $x \in M_{\delta}$ и $X \in T_{x} M_{\delta}$ положим

$$
\sigma(X)=\int_{0}^{\delta} h\left(\frac{\nabla}{d t}\left(\frac{d x}{d t}\right)+F^{\#}\left(\frac{d x}{d t}\right)+\operatorname{grad} u, X\right) d t
$$

Этим определена замкнутая 1-форома $\sigma$ на $M_{\delta}$.

Если $\Pi=\pi_{1}(B, b) \neq 0$, то многообразие $M_{\delta}$ несвязно. Выберем компоненту связности $D \in \pi_{0}\left(M_{\delta}\right)$ и зафиксируем “опорньй" путь $x_{D} \in D$. Символом $\widehat{D}$ обозначим многообразие кусочно гладких гомотопий $c:[0, \delta] \times I \rightarrow B$, удовлетворяющих условиям $c(t, 0)=x_{D}(t), c(0, \tau) \equiv a$ и $c(\delta, \tau) \equiv b$. Для каждого $\tau \in I$ положим $c_{\tau}(t)=c(t, \tau)$. Тогда $c_{\tau} \in D$ и $c_{0}=x_{D}$. Две гомотопии $c, c^{\prime} \in \widehat{D}$ будем считать эквивалентными при $c_{1}=c_{1}^{\prime}$ и $I_{[\sigma]}\left(\left[c^{\prime}-c\right]\right)=0$. Соответствующее фактормногообразие обозначим символом $\widetilde{D}$. Определим регулярное накрытие $\mu: \widetilde{D} \rightarrow D$ формулой $\mu([c])=c_{1}$, где $[c]-$ класс эквивалентности гомотопии $c \in \widehat{D}$. Рассмотрим класс $[c] \in \widetilde{D}$, путь $x=c_{1}$ и положим

$$
S(x)=\int_{0}^{\delta}\left(\frac{h(d x / d t, d x / d t)}{2}+u(x)\right) d t+\int_{c} F .
$$

Тогда $S: \widetilde{D} \rightarrow \mathbb{R}$ - гладкая функция и $d S=\mu^{*} \sigma$. Согласно [4] $S$ - многозначный функционал на $D$. Пусть $[c]$ - экстремум функции $S: \widetilde{D} \rightarrow \mathbb{R}$. Тогда путь $x=c_{1}$ естественно называть экстремалью многозначного функционала $S$ на $D$ или соответствуюшей формы $\sigma$. В случае, когда $h$ - риманова метрика, экстремали формы $\sigma$ являются движениями натуральной гироскопической системы $Г$ из положения $a$ в положение $b$ за время $\delta$. Если $h$ - лоренцева метрика и $u \equiv 1 / 2$, то $\Gamma$ - гироскопическая система релятивистского типа. Ее движениями мы считаем экстремали $x \in D$ формы $\sigma$, удовлетворяющие тождеству

$$
h\left(\frac{d x}{d t}, \frac{d x}{d t}\right) \equiv-1
$$

В теории относительности они интерпретируются как мировые линии заряженных пробных частиц в гравитационном поле с потенциалом $h$ и электромагнитном поле с формой $F$. 
Заметим, что при $[\sigma] \neq 0$ в $H^{1}(D, \mathbb{R})$ имеет место равенство $\mathrm{im} S=\mathbb{R}$. Поэтому даже для вывода условий существования экстремалей многозначного функционала $S$ стандартные методы вариационного исчисления неприменимы.

По построению $\widetilde{D} \approx D$ в том и только том случае, если $I_{[\sigma]}\left(\left[c^{\prime}-c^{\prime \prime}\right]\right)=0$ для любых гомотопий $c^{\prime}, c^{\prime \prime} \in \widehat{D}$ с $c_{1}^{\prime}=c_{1}^{\prime \prime}$. Последнее равносильно справедливости равенства $\int_{c} F=0$ для произвольного двумерного сфероида $c$ в $B$. С другой стороны, $[\sigma]=0$ тогда и только тогда, когда $\widetilde{D} \approx D$. Следовательно, препятствием к однозначности функционала $S$ является факторгруппа im $\chi / \operatorname{ker} J_{[F]}$, где $\chi: \pi_{2}(B, b) \rightarrow H_{2}(B)$ - гомоморфизм Гуревича, а $J_{[F]}$ - сужение гомоморфизма $I_{[F]} \in \operatorname{Hom}\left(H_{2}(B), \mathbb{R}\right)$ на его образ im $\chi$.

Пусть $\Lambda$ - нормальньй делитель группы $\Pi=\pi_{1}(B, b)$. Рассмотрим неотрицательные целые числа $k, l$ и $m=k+l$. Предположим, что форма $F$ имеет представление

$$
F=\theta \circ \Phi, \quad \theta \in \operatorname{Hom}\left(\mathbb{R}^{m}, \mathbb{R}\right), \quad[\Phi] \in H^{2}\left(B\left|\Lambda, \mathbb{R}^{m}\right| \mathbb{Z}^{k}\right)
$$

Отметим, что условие (7.1) заведомо выполнено в случае, когда подгруппа $H_{2}(B \mid \Lambda) \subset H_{2}(B)$ конечно порождена. В этой ситуации число $k$ может быть выбрано не превьшающим величины $\operatorname{rank} H_{2}(B \mid \Lambda)$. Выбор числа $l$ вообще произволен. Добавим, что наименњшее допустимое значение параметра $m$ равно $k$ при $l=0$ и $\operatorname{ker} \theta \cap \mathbb{Z}^{k}=0$.

Положим $\Delta=\Pi / \Lambda, T=T^{k} \times \mathbb{R}^{l}$ и $G=\Delta \times T$. Тогда согласно предложению 3.2 существует почти главное $G \mid \Lambda$-расслоение $\rho=(E, p, B, \mathscr{A})$ с характеристическим $I$-классом $[\Phi]$. На пространстве $E$ имеется $G$-связность $H$ с формой связности $\omega$ и формой кривизны $d \omega=p^{*} \Phi$. Поэтому $p^{*} F=d(\theta \circ \omega)$.

Рассмотрим стандартный базис $e_{1}, \ldots, e_{m}$ пространства $\mathbb{R}^{m}$ и дуальный к нему базис $e^{1}, \ldots, e^{m}$ сопряженного пространства $\left(\mathbb{R}^{m}\right)^{*}=\operatorname{Hom}\left(\mathbb{R}^{m}, \mathbb{R}\right)$. Формула $j\left(r_{1} e^{1}+\cdots+r_{m} e^{m}\right)=r_{1} e_{1}+\cdots+r_{m} e_{m}$ определяет изоморфизм $j: \operatorname{Hom}\left(\mathbb{R}^{m}, \mathbb{R}\right) \rightarrow$ $\mathbb{R}^{m}$. Положим $\theta^{2}=\theta(j(\theta)), \mathscr{Q}=j(\theta) / \theta^{2}$ и $\gamma(r, s)=\theta^{2}\left(r_{1} s_{1}+\cdots+r_{m} s_{m}\right)$ для $r, s \in \mathbb{R}^{m}$. Тогда $\gamma(\mathscr{Q}, r)=\theta(r)$ и $\gamma(\mathscr{Q}, \mathscr{Q})=\theta(\mathscr{Q})=1$. Таким образом, $\mathscr{Q}-$ орт евклидова пространства $\left(\mathbb{R}^{m}, \gamma\right)$, ортогональньй подпространству $\operatorname{ker} \theta$. Пусть $p_{\theta}(r)=r-\theta(r) \mathscr{Q}$ для $r \in \mathbb{R}^{m}$ и $\Theta=e(\operatorname{ker} \theta)$. Этим определены ортогональная проекция $p_{\theta}: \mathbb{R}^{m} \rightarrow \operatorname{ker} \theta$ и $(m-1)$-мерная подгруппа $\Theta$ группы $T$.

Допустим, что $u>0$. Положим $u^{*}=u \circ p, \gamma^{u}=\gamma / 2 u^{*}, \omega^{u}=\omega / 2 u^{*}$,

$$
\mathscr{H}_{v}=\left\{X \in T_{v} E: p_{\theta} \circ \omega(X)=0\right\}, \quad g(X, Y)=\gamma^{u}(\omega(X), \omega(Y))+p^{*} h(X, Y)
$$

для любых $v \in E$ и $X, Y \in T_{v} E$. Тогда $\omega^{u}-\mathbb{R}^{m}$-значная 1-форма на $E, \mathscr{H}: v \rightarrow \mathscr{H}_{v}$ - гладкое распределение размерности $\operatorname{dim} B+1$ и $g$ - псевдориманова метрика на $E$. По построению метрика $g$ инвариантна относительно действия групшы $T$ и локальных действий группы $\Delta$ и невырождена на слоях расслоения $\rho$. В такой ситуации мы называем $(E, g)$ многообразием Калуцы-Клейна.

Подмногообразия $\mathscr{F}(v)=\{v \cdot t, t \in \Theta\}, v \in E$, образуют на $E$ риманово слоение $\mathscr{F}$ коразмерности $\operatorname{dim} B+1$. При этом $g$-метрика Рейнхарта, а $\mathscr{H}$ - ортогональная слоям связность Эресмана для $\mathscr{F}$. 
Пусть $y: I \rightarrow E-$ кусочно гладкий путь. Положим $x=p \circ y$ и рассмотрим горизонтальный лифт $y^{0}$ пути $x$ с началом $y^{0}(0)=y(0)$ относительно $G$-связности $H$. Поскольку $p \circ y=p \circ y^{0}$, то найдется, причем единственный, кусочно гладкий путь $z: I \rightarrow \mathbb{R}^{m}$, удовлетворяющий равенствам $z(0)=0$ и $y(s)=y^{0}(s) \cdot e(z(s))$. Мы говорим, что путь $y$ увеличивает форму $\theta \in \operatorname{Hom}\left(\mathbb{R}^{m}, \mathbb{R}\right)$, если $\theta(z(1))>0$.

Для числа $\delta \in \mathbb{R}_{+}=(0,+\infty)$, пути $x \in M_{\delta}$ и точки $v \in G_{a}$ сушествует единственный кусочно гладкий путь $y: I \rightarrow E$ с началом $y(0)=v$, горизонтальный относительно связности Эресмана $\mathscr{H}$ и удовлетворяющий равенствам $\theta \circ \omega^{u}\left(\frac{d y}{d s}\right) \equiv \delta$ и $p \circ y\left(\frac{t}{\delta}\right)=x(t)$. При этом мы полагаем $y=\lambda^{v}(x)$.

Пути $x \in M_{\delta}$ и $x^{*} \in M_{\delta^{*}}$ будем считать $\Lambda$-эквивалентными, если $\left[x_{1}+x_{1}^{*}\right] \in \Lambda$ для путей $x_{1}(s)=x(s \delta)$ и $x_{1}^{*}(s)=x^{*}\left(s \delta^{*}\right), s \in I=[0,1]$. Каждому классу $\Lambda$-эквивалентности $C$ из объединения $M=\bigcup_{\delta \in \mathbb{R}_{+}} M_{\delta}$ соответствует компонента связности $T_{C}$ слоя $G_{b}=p^{-1}(b)$, содержащая конщы путей $y=\lambda^{v}(x)$ для всех $x \in C$. Символом $\Omega\left(v, T_{C}\right)$ обозначим пространство кусочно гладких путей $y: I \rightarrow E$ с началом $y(0)=v$ и концом $y(1) \in T_{C}$.

Теорема 7.1. Пусть $h$ - риманова метрика. Тогда отображсене $\lambda^{v}: C \rightarrow$ $\Omega\left(v, T_{C}\right)$ устанавливает биективное соответствие межсду множеством принадлежсащих $\Lambda$-классу $C$ движений натуральной гироскопической системы Г и множсеством геодезических риманова многообразия Калуцы-Клейна $(E, g)$, принадлежсащи пространству $\Omega\left(v, T_{C}\right)$, ортогональных слоям слоения $\mathscr{F}$ и увеличивающих форму $\theta$. Если $h$-лоренцева метрика, то $\lambda^{v}$ задает биекиию множества принадлежащих $C$ движений гироскопической системь релятивистского типа на множсетво обладающих теми же свойствами изотропных геодезических лоренцева многообразия $(E, g)$.

Формально теорема 7.1 является обобщением теоремы 1 из [5] и теоремы 2 из [6], в которых использованы почти главные расслоения с инвариантом $\Lambda=0$. Однако ее обоснование, по существу, сводится к повторению доказательств обобщаемых теорем и потому здесь опускается.

Ортогональность геодезической $y: I \rightarrow E$ слоям риманова слоения $\mathscr{F}$ во всех точках равносильна ее ортогональности одному слою $L \in \mathscr{F}$, проходящему через концевую точку $y(1)$. Поэтому теорема 7.1 сводит изучение принадлежащих классу $C$ движений гироскопической системы $\Gamma$ к вариационной задаче для функционала длины псевдориманова многообразия Калуцы-Клейна $(E, g)$ на пространствах вида $\Omega(v, L)$. Последняя является почти классической задачей с фиксированным началом и подвижным концом для однозначного и положительного функционала. Некоторая ее нестандартность состоит лишш в том, что концевые подмногообразия - слои слоения $\mathscr{F}$ - могут быть некомпактными и даже несобственными. Требование роста значений формы $\theta$ вдоль экстремалей модельной задачи накладывает ограничение на выбор лежащих в $T_{C}$ концевых подмногообразий.

Так как $H_{2}(B \mid 0) \subset H_{2}(B \mid \Lambda) \subset H_{2}(B \mid \Pi)=H_{2}(B)$ для всех нормальных делителей $\Lambda \subset$ П, то при $\Lambda=$ П условие (7.1) на форму гироскопических сил $F$ оказывается наиболее обременительным, а допустимая размерность структурной группы 
почти главного расслоения $\rho$ максимальна. Кроме того, в рассматриваемой ситуации имеется единственный класс $\Lambda$-эквивалентности $C=M$. Ему соответствует единственная компонента связности $T_{C}=G_{b}$ слоя над точкой $b$. Поэтому редукция теоремы 7.1 не позволяет различать не гомотопные движения системы Г из точки $a$ в точку $b$. Таким образом, использование почти главных $G \mid \Pi$-расслоений - главных расслоений со структурной группой $T$ - в двухконцевой задаче для многозначных функционалов оказьвается наименее выгодным.

Выбор инварианта $\Lambda$ равньп нулю оптимален по ряду причин. Во-первых, при этом мы имеем самое слабое ограничение на форму $F$ и, следовательно, наибольшую область применимости обсуждаемого метода исследования динамики гироскопических систем. Во-вторых, оказывается минимальной размерность структурной группы используемого почти главного $G \mid \Lambda$-расслоения, которая явным образом входит в формулировки полученных этим методом результатов [5], [6]. В-третьих, $\Lambda$-эквивалентные пути из $M$ при $\Lambda=0$ гомотопны. Поэтому с помощью редукции теоремы 7.1 могут быть получены теоремы существования движений гироскопической системы Г, принадлежащих заданному гомотопическому классу идущих из $a$ в $b$ путей. Наконец, как мы отмечали выше, многозначность функционала $S$ - действия системы $\Gamma$ - связана с поведением формы гироскопических сил только на образе гомоморфизма Гуревича $\chi: \pi_{2}(B, a) \rightarrow H_{2}(B)$, а не на всей группе гомологий $H_{2}(B)$. Логично предположить, что и особенности вариационной задачи для многозначных функционалов определяются гомоморфизмом $J_{[F]} \in \operatorname{Hom}(\operatorname{im} \chi, \mathbb{R})$. Следовательно, для выявления таких особенностей целесообразно использование почти главных расслоений, инварианты которых связаны с подгруппой $\operatorname{im} \chi \subset H_{2}(B)$. Осталось заметить, что $H_{2}(B \mid \Lambda)=\operatorname{im} \chi$ тогда и только тогда, когда $\Lambda=0$. Кстати, при $\Pi \neq 0$ почти главные $G \mid 0$-расслоения наиболее сильно отличаются от главных $T$-расслоений.

Упомянутое в предыдущем абзаце предположение не только логично, но и основано на конкретных примерах. Рассмотрим в трехмерном евклидовом пространстве поверхности $B_{1}=T^{2}$ и $B_{2}=S^{2}$. Пусть $h_{i}$ - индуцированные римановы метрики на $B_{i}, F_{i}$ - их нормированные формы плошади, $u_{i} \equiv$ const, $i=1$, 2 . Тогда оба гомоморфизма $I_{\left[F_{i}\right]}: H_{2}\left(B_{i}\right) \rightarrow \mathbb{R}$ удовлетворяют равенствам $\operatorname{im} I_{\left[F_{i}\right]}=\mathbb{Z}$, т.е. поведение форм $F_{i}$ на группах гомологий $H_{2}\left(B_{i}\right) \cong \mathbb{Z}$ одинаково. Однако если для любых точек $a_{1}, b_{1} \in B_{1}$ в каждом гомотопическом классе идущих из $a_{1}$ в $b_{1}$ путей содержатся движения гироскопической системы $\Gamma_{1}=\left(B_{1}, h_{1}, F_{1}, u_{1}\right)$, то для диаметрально противоположных точек $a_{2}$ и $b_{2}$ сферы $B_{2}$ ни одного движения системы $\Gamma_{2}=\left(B_{2}, h_{2}, F_{2}, u_{2}\right)$ из $a_{2}$ в $b_{2}$ быть не может. Следовательно, между рассматриваемыми двумя системами должно существовать принципиальное различие. Для его обнаружения достаточно заметить, что гомоморфизм Гуревича $\chi_{i}: \pi_{2}\left(B_{i}, a_{i}\right) \rightarrow H_{2}\left(B_{i}\right)$ при $i=1$ тривиален, а при $i=2$ является изоморфизмом. Поэтому $J_{\left[F_{1}\right]}=0$, a im $J_{\left[F_{2}\right]}=\operatorname{im} I_{\left[F_{2}\right]}=\mathbb{Z}$.

\section{Список литературы}

1. Kobayashi S. Principal fibre bundles with the 1-dimensional toroidal group // Tôhoky Math. J. 1956. V. 8. P. 29-45.

2. Kodaira K., Spenser D. S. Groups of complex line bundles over compact Kähler manifolds // Proc. Nat. Acad. Aci. U.S.A. 1953. V. 39. P. 868-872. 
3. Хьюзмоллер Д. Расслоенные пространства. М.: Мир, 1970.

4. Новиков С. П. Гамильонов формализм и многозначный аналог теории Морса // УМН. 1982. Т. 37. № 5. С. 3-49.

5. Яковлев Е. И. Геодезическое моделирование и условия разрешимости двухконщевой задачи для многозначных функционалов // Функц. анализ и его прилож. 1996. Т. 30. № 1 . C. $89-92$.

6. Яковлев Е.И. О существовании решений двухточечных краевых задач для гироскопических систем релятивистского типа // Алгебра и анализ. 1997. Т. 9. № 2. С. 256-271.

Нижегородский государственный университет

E-mail: yakovlev@mm.unn.ac.ru
Поступила в редакцию 22.07 .1998 и 08.06 .1999 